九州大学学術情報リポジトリ

Kyushu University Institutional Repository

\title{
Theoretical stability of the polarization in a thin semiconducting ferroelectric
}

Watanabe, Yukio

Kyushu Institute of Technology

http://hdl. handle. net/2324/4354933

出版情報: Physical review B. 57 (2)，pp.789-804，1998-01-01. American Physical Society バージョン:

権利関係: (C) 1998 The American Physical Society 


\title{
Theoretical stability of the polarization in a thin semiconducting ferroelectric
}

\author{
Yukio Watanabe \\ Kyushu Institute of Technology, Kitakyusyu, Fukuoka 804, Japan
}

(Received 7 May 1997)

\begin{abstract}
The size effect in ferroelectrics is examined by considering the semiconductivity of the ferroelectric. This approach is applied to a theoretical investigation of the stability of the spontaneous polarization in a ferroelectric film on a semiconductor using a homogeneous Ginzburg-Landau theory. The band structure in the ferroelectric/insulator/semiconductor is rigorously incorporated in the theory, as if for a conventional semiconductor heterostructure such as $\mathrm{GaAs} /(\mathrm{Ga}, \mathrm{Al}) \mathrm{As}$. The carriers generated in the ferroelectric are found to change drastically the stability of the spontaneous polarization and its size effect. Numerical and simple analytical results are presented for $\mathrm{BaTiO}_{3}$ and, mostly, for $\mathrm{PbTiO}_{3}$. The spontaneous polarization in a single-domain ferroelectric on a semiconductor is shown to be bistable and induce a semiconductor space charge $D$. The value of $D$ is a tiny fraction of the spontaneous polarization and agrees with experimentally estimated values. Namely, the experimental quasiequilibrium $D$ values are mostly intrinsically limited by the heteroband structure, not by the technological difficulties. Moreover, the $D$ value for a finite insulator thickness was found to be mainly determined by the insulator thickness and the semiconductor properties of the ferroelectric, and was relatively insensitive to the ordinary ferroelectric properties and the trap densities at the interface. The theory forecasts possible limitations of the transistors using this structure. Nonetheless, a long retention of the semiconductor space-charge $D$ by a very thin ferroelectric can be realized by the advancement of the technology. Additionally, the stability of very thin ferroelectrics on semi-insulating substrates and the switching of ferroelectrics on parent materials of the high- $T_{c}$ superconductors are successfully explained.
\end{abstract}

[S0163-1829(97)07446-8]

\section{INTRODUCTION}

Mathematical treatment of ferroelectricity is similar to that of ferromagnetism in many respects including multidomain structures that reduce the depolarization or the demagnetization field. However, the existence of the electron and the absence of magnetic monopoles allows a treatment of the depolarization different from that of the demagnetization fields. The free-carrier effect in narrow-gap ferroelectrics has been theoretically studied to understand the bulk ferroelectricity in these materials. ${ }^{1}$ On the other hand, a space-charge layer at the ferroelectric surface has been suggested. ${ }^{2,3}$ The surface layer would modify the ferroelectric size effect and also the performances of ferroelectric thin-film devices. However, its nature has been relatively unknown until now. A tentative model of the surface layer is the gradual change of the spontaneous polarization $P_{s}$ near the surface as proposed for ferromagnetism and can work without a free carrier. $^{4-7}$ For displacive-type ferroelectriclike $\mathrm{BaTiO}_{3}$ and $\mathrm{PbTiO}_{3}$, this means that the crystal structure changes gradually near their surfaces. On the other hand, the surface layer can also be a chemically and crystallographically disordered layer due to sample preparation. Alternatively, it can be a relatively abrupt change of crystal structure due to sample preparation such as an inadequate oxygenation.

However, the advancement of the thin-film technology has removed such extraneous surface effects by supplying epitaxial single-crystal-like films. In such films, disorders at the surface and the interface are substantially eliminated. For example, the crystallographic structure of $\mathrm{BaTiO}_{3}$ was found to be that of a ferroelectric phase, even at the thickness of 14 nm. ${ }^{8}$ Moreover, crystallographic parameters of the $\mathrm{BaTiO}_{3}$ on a $\mathrm{SrTiO}_{3}$ (insulator) is the same as those on a Nb-doped $\mathrm{SrTiO}_{3}$ (conductor). ${ }^{8}$ Furthermore, a freestanding $10 \mathrm{~nm}$ thick $\mathrm{PbTiO}_{3}$ platelet exfoliated from an epitaxial film has the crystallographic symmetry of the ferroelectric phase. ${ }^{9}$ These observations are puzzling, if we consider the enormous depolarization field.

The ferroelectric/semiconductor $(\mathrm{F} / \mathrm{S})$ structure has been pursued toward realization of a ferroelectric field-effect device. ${ }^{10-12}$ Recently, the performances of a modified F/S are reported that employ an insulator between the ferroelectric and the semiconductor (F/I/S). These experimental results are again difficult to understand in view of a conventional depolarization field instability as discussed in a separate paper. ${ }^{13}$ Additionally, the ferroelectric switching was observed in a parent phase of a high- $T_{c}$ superconductor, e.g., $\mathrm{La}_{2} \mathrm{CuO}_{4},{ }^{14}$ while it is not yet reported to have a possibility of the electron donor doping. This means that $\mathrm{La}_{2} \mathrm{CuO}_{4}$ may not supply any electron to compensate the ferroelectric charge and thus act as an insulator when the ferroelectric polarization is directed toward $\mathrm{La}_{2} \mathrm{CuO}_{4}$. In this case, the ferroelectric phase should be unstable, if the ferroelectric is insulating and does not have enough electronic states to accommodate electrons.

Ferroelectrics like $\mathrm{PbTiO}_{3}$ have usually been regarded as insulators, when their ferroelectric properties are discussed. A few Russian physicists have theoretically investigated a $p n$ junction in ferroelectrics and the field effect in the $\mathrm{F} / \mathrm{S}$ structures. ${ }^{15,16}$ Although their results were difficult to compare with experiments so far, the latter was developed into the depolarization instability by Batra and co-workers. ${ }^{17,18}$

The viewpoint regarding ferroelectrics as a semiconductor 
seems to be confirmed by recent experiments. For example, a space-charge layer at metal/ferroelectric contact was suggested by the current through ferroelectric/metal structures, which showed Schottky-like conduction. ${ }^{19}$ Similar phenomena were also observed in epitaxial $\mathrm{F} / \mathrm{S}$ heterostructures. ${ }^{20,21}$ Similarly, the space-charge effect, i.e., Schottky barrier at the metal/ferroelectric interface is considered to be one of the mechanisms that intrinsically reduce the dielectric constant of very thin high-dielectric films like $\mathrm{SrTiO}_{3}$ and $\mathrm{Pb}(\mathrm{Zr}, \mathrm{TiO}) \mathrm{O}_{3}$.

The discussions above also suggest a crucial effect of electrodes on ferroelectric properties, especially on very thin/ small ferroelectrics. Therefore, we need to specify the nature of the electrode or the type of the heterostructures, even when we discuss an intrinsic nature of ferroelectrics, because most electrical measurements use electrodes or contacts between different particles. For example, metallic electrodes can kill the depolarization effect, especially in a multidomained ferroelectric.

Ginzburg-Landau-Devonshire (GLD) thermodynamic theory has been frequently used to analyze properties of both bulk and small-scale ferroelectrics. Ferroelectrics free of interfacial defects and disorders can be prepared due to the recent advancement of material technology. Therefore, we may meaningfully analyze single crystalline ferroelectric thin films of which the top surface has the same crystallographic quality, or atomic-bond energies, as that inside. For such a small ferroelectric, the application of GLD theory is allowed, because thermodynamic fluctuations are important only in a particulate having a diameter of a few $\mathrm{nm}$ or less. $^{22,23}$

Encouraged by these results, we investigated the stability of the ferroelectric polarization in typical ferroelectric heterostructures having various thicknesses, by incorporating semiconductive properties into a GLD theory. Here, a very low mobility of the free carrier in the ferroelectric does not matter. The approach in the present paper is similar to Ref. 13 , except for the rigorous treatment of the semiconductivity of the ferroelectric. Namely, we consider the stability of spontaneous polarization $P_{S}$ in a unidomain (single domain), assuming a homogeneous $P_{s}$ in the ferroelectric, i.e., $\nabla P \sim 0$. Here, a "unidomain" means that the width of the domains of, at least, one polarity is much larger than the ferroelectric thickness and the space-charge layer thickness.

By incorporating the effect of the multidomain and the inhomogeneity $(\nabla P)$, we can further decrease the minimum GLD energy, because it gives more free parameters. Therefore, we do not expect that $\nabla P=0$ and a unidomain occurs absolutely in a real ferroelectric film (Appendix A). However, our point is that these two effects are not as essential as the other properties like semiconductivity in ferroelectrics, due to a large depolarization field. Additionally, the carriers in the ferroelectric and the semiconductor are treated nonquantum mechanically, and Boltzmann distribution is used. These assumptions are examined in Ref. 13, and, especially, the effect of multidomain was found to be insufficient to stabilize the ferroelectric phase in a thin film of a thickness less than $200 \mathrm{~nm}$.

Additionally, several experimental results suggest the existence of unidomainlike structures. One of them is the crystallographic symmetry and parameters of very thin ferroelec- tric films. Secondly, stable poled regions having a lateral dimension of a few micrometers are observed by a scanning probe method in a ferroelectric film formed on a bottom metal electrode. ${ }^{24}$ The regions appear to be unidomain, although this structure is regarded as ferroelectric/insulator (=air). Thirdly, F/I/S structures exhibited a ferroelectric field effect modulation of the semiconductor conductance, although it was reported to disappear in a short period. ${ }^{11,12}$ The thickness and the lateral dimension of the ferroelectric gate was typically $300 \mathrm{~nm}$ and $10 \mu \mathrm{m}$, respectively. This indicates that the size of domains having one polarity increased and that with the opposite decreased. Furthermore, the large modulation ratio and the high conductance in the on state would be difficult to explain, if there were many $p n$ junctions under the ferroelectric gate. Similar results on the capacitance-voltage characteristics are discussed in Appendix B.

Below, the $c$ axis of the ferroelectrics, i.e., the direction of $P_{s}$, is assumed to be perpendicular to the film surface. This is because the result for the ferroelectric with the $c$ axis parallel to the film surface is trivial and the result with the $c$ axis perpendicular to the film surface is most drastically changed by the depolarization instability.

The present approach shows a drastic effect of ferroelectric carriers, especially on the ferroelectric on the insulator and $\mathrm{F} / \mathrm{I} / \mathrm{S}$ and on the thickness dependence, and explains several experimental results. It also demonstrates the effect of the trap or acceptor centers in the ferroelectric and at its interface. The subsequent part of the paper is organized as follows: formulation and approximate analytical results (Sec. II), numerical results (Sec. III), and discussion and comparison with experiments (Sec. IV).

\section{FORMULATION AND SOLUTION}

\section{A. Formulation}

We consider the structure as in Fig. 1, where $l_{f}$ and $l_{d}$ are the thickness of the ferroelectric and the insulating layer, respectively. The $x$ coordinate is defined in the direction perpendicular to the film, where $x=0$ is at the boundary between the insulator and the semiconductor. Here, we do not include the effect of the disorder and defects at the interface, the effect of which is discussed in Sec. IV. The F/S structure represents an artificially formed F/S as well as the ferroelectric having a dead layer or an amorphous layer that lost the ferroelectricity. Additionally, a F/S with a sufficiently large semiconductor band gap can represent a ferroelectric/semiinsulator structure. The formulation in this subsection is similar to that in Ref. 13, where more details of the specific issues are described. The present result is applicable to a ferroelectric particulate by regarding it as $I / F / I$ and $l_{f}$ as half of its thickness.

For a $\mathrm{F} / \mathrm{I} / \mathrm{S}$, the polarization $(P)$ dependent part of free energy density $F$ (per $\mathrm{cm}^{3}$ ) is written as

$$
F=F_{f}(P)+F_{d}(P) / l_{f}+F_{s}(P) / l_{f},
$$

where $F_{f}, F_{d}$, and $F_{s}$ correspond to the free energy density of the ferroelectric and the free energies per area of the insulator and the semiconductor, respectively. According to Appendix $\mathrm{C}, F_{f}$ for $\mathrm{BaTiO}_{3}$ and $\mathrm{PbTiO}_{3}$ is written as 


$$
\begin{gathered}
F_{f}=F_{0}+\eta(\nabla P)^{2}+\varepsilon_{0} \int_{-l_{f}-l_{d}}^{-l_{d}} d x E_{f}^{2} / 2 l_{f} \\
-P\left(\delta \phi+V_{e}\right) / l_{f} \\
F_{0}=\alpha_{1} P^{2}+\alpha_{11} P^{4}+\alpha_{111} P^{6},
\end{gathered}
$$

where $E_{f}$ is the electric field in the ferroelectric and $F_{0}$ is the free energy of a bulk ferroelectric, $\delta \phi$ is the work function difference, and $V_{e}$ is the external field. The inverse of the linear susceptibility $\alpha_{1}$ is written as $\alpha_{1}=(T-\theta) / 2 \varepsilon_{0} C$, where $\theta$ and $T$ are the Curie temperature $\left(T_{c}\right)$ and the ambient temperature, respectively. For $\nabla P \neq 0, F_{f}$ should be integrated from $x=-l_{f}-l_{d}$ to $x=-l_{d}$ and then divided by $l_{f}$. In Eq. (2), we did not include the stress terms in GLD theory. A total free energy for $\delta \phi+V_{e}=0$ including the stress is

$$
\begin{aligned}
F_{f}= & \alpha_{1} P^{2}+\alpha_{11} P^{4}+\alpha_{111} P^{6}+\eta(\nabla P)^{2} \\
& +\varepsilon_{0} \int_{-l_{f}-l_{d}}^{-l_{d}} d x E_{f}^{2} / 2 l_{f}+F_{s 1}(\underline{S}) / 2 \varepsilon_{0} P^{2}+F_{s 2}(\underline{S}) \\
= & \left\{T-\left[\theta-C F_{s 1}(\underline{S})\right]\right\} / 2 \varepsilon_{0} C P^{2}+\alpha_{11} P^{4}+\alpha_{111} P^{6} \\
& +\eta(\nabla P)^{2}+\varepsilon_{0} \int_{-l_{f}-l_{d}}^{-l_{d}} d x E_{f}^{2} / 2 l_{f}+F_{s 2}(\underline{S}),
\end{aligned}
$$

where $F_{s 1}(\underline{S})$ and $F_{s 2}(\underline{S})$ are functions only dependent on the stress tensor $(\underline{S}) .^{25,26}$ For $c$-axis oriented ferroelectric films, Eq. (2b) indicates that the effect of the stress is only to change an effective Tc and the zero-point energy, i.e., the energy at $P=0$. A numerical analysis using Eq. (2b) shows that this effect is small as compared with the depolarization energy in the absence of the free carrier. ${ }^{27}$ Furthermore, it can be minimized by selecting a material and the preparation processes. Therefore, the neglect of the stress term is inessential.

$F_{d}$ and $F_{s}$ are written as

$$
\begin{gathered}
F_{d}=l_{e} D^{2} / 2 \varepsilon_{0}, \\
F_{s}=\varepsilon_{s} \varepsilon_{0} \int_{0}^{\infty} d x E(x)^{2} / 2,
\end{gathered}
$$

where $\varepsilon_{0}$ is the vacuum permittivity, $\varepsilon_{s}$ is the dielectric constant of the semiconductor, $E(x)$ is the electric field in the semiconductor, $D$ is the electric flux (displacement vector), and $l_{e}$ is the effective thickness of the insulator defined below in Eq. (5),

$$
l_{e}=l_{d} / \varepsilon_{d},
$$

where $\varepsilon_{d}$ is the dielectric constant of the insulator. Equation (5) can be easily extended to a multilayered intermediate layer (Ref. 13).

$D$ and $E_{s} \equiv E(0)$ are mutually related via Gauss's law at the insulator/semiconductor (I/S) and the ferroelectric/ insulator $(\mathrm{F} / \mathrm{I})$ boundaries,

$$
\begin{aligned}
D & =\varepsilon_{s} \varepsilon_{0} E_{s} \quad \text { at } x=0 \\
& =\varepsilon_{0} E_{f}+P \quad \text { at } x=-l_{d} .
\end{aligned}
$$
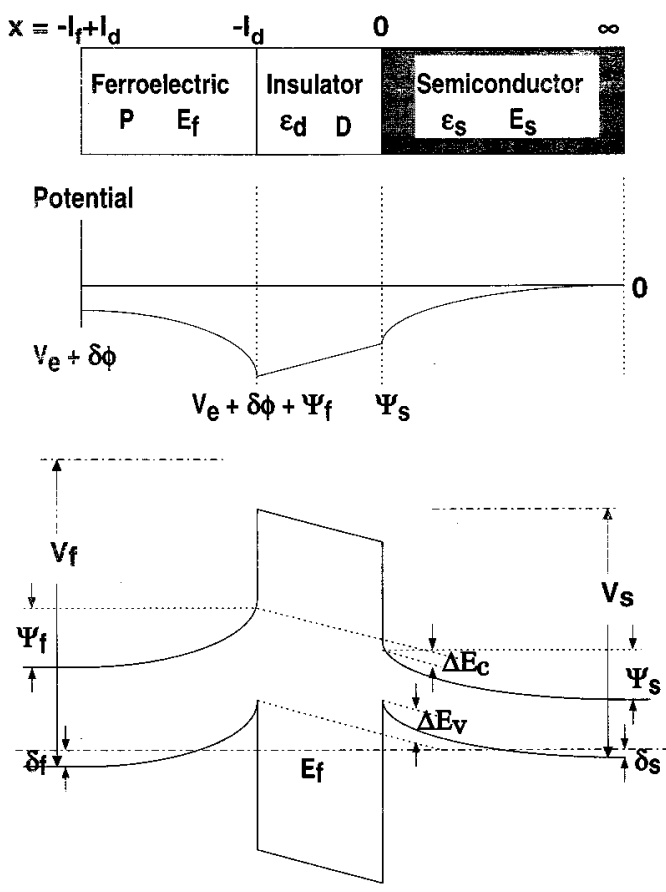

FIG. 1. F/I/S (ferroelectric/insulator/semiconductor) structure, the distribution of potential, the heteroband structure.

Using the surface potentials $\Psi_{s} \equiv \Psi_{s}(D) \equiv \Psi(x=0)$ and $\Psi_{f} \equiv \Psi_{f}(D) \equiv \Psi\left(x=-l_{d}\right)-\delta \phi-V_{e}$, we have an equation for the potential (Fig. 1),

$$
\Psi_{s}(D)+l_{e} D / \varepsilon_{0}=\Psi_{f}(D)+\delta \phi+V_{e} .
$$

The bottom of the semiconductor is grounded. We incorporated $\delta \phi$ in the theory, following the scheme used in metaloxide-semiconductor (MOS) devices. ${ }^{28,29}$

For a given semiconductor material parameter, the doping level, and the temperature, the relationship between $E_{s}$ and $\Psi_{s}$ is given by solving the Poisson equation, ${ }^{29}$

$$
-\Delta \Psi(x)=e\left(N_{D}^{+}-N_{A}^{-}+p-n\right) / \varepsilon_{s} \varepsilon_{0},
$$

where $e$ is the elementary charge, and $N_{D}^{+}, N_{A}^{-}, p$, and $n$ are the donor density, the acceptor density, the hole carrier density, and the electron density in the semiconductor, respectively.

Similarly, the relationship between $E_{f}$ and $\Psi_{f}$ is given by solving the Poisson equation for $\nabla P=0$,

$$
-\Delta \Psi(x)=e\left(N_{D f}^{+}-N_{A f}^{-}+p_{f}-n_{f}\right) / \varepsilon_{0},
$$

where $N_{D f}^{+}, N_{A f}^{-}, p_{f}$, and $n_{f}$ are the donor density, the acceptor density, the hole carrier density, and the electron density in the ferroelectric, respectively.

Equations (2)-(8) form the basic equations in this theory.

Assuming Boltzmann statistics, Eq. (8a) is rewritten as

$$
\begin{aligned}
\Delta \Psi(x)= & e\left(p_{0}\{\exp [-\beta \Psi(x)]-1\}\right. \\
& \left.-n_{0}\{\exp [\beta \Psi(x)]-1\}\right) / \varepsilon_{s} \varepsilon_{0},
\end{aligned}
$$

where $\beta=e / k T, k$ is the Boltzmann constant, $T$ is the temperature, and $p_{0}$ and $n_{0}$ are the hole carrier density and the electron destiny at $x=\infty$, i.e., at $\Psi=0$. In the following ar- 
gument, $x>100 \mathrm{~nm}$ is regarded as $\infty$, when $\Psi_{s}$ is of order of $1 \mathrm{~V}$. The intrinsic carrier density $n_{i}, p_{0}$, and $n_{0}$ at $T$ are given by ${ }^{29}$

$$
\begin{gathered}
p_{0}=\left\{N_{A}-N_{D}+\left[\left(N_{A}-N_{D}\right)^{2}+4 n_{i}^{2}\right]^{1 / 2}\right\} / 2, \\
n_{0}=n_{i}^{2} / p_{0}, \\
n_{i} / n_{i}\left(T_{0}\right)=\left(T / T_{0}\right)^{3 / 2} \exp \left[-E g / 2 k T-E g\left(T_{0}\right) / 2 k T_{0}\right],
\end{gathered}
$$

where $E g$ is the band gap of the semiconductor at $T$, and $E g\left(T_{0}\right)$ and $n i\left(T_{0}\right)$ are the band gap and the intrinsic carrier density at $T=T_{0}$, e.g., $300 \mathrm{~K}$, respectively. The temperature dependence of $E g$ is given by

$$
E g=E g(0)-\gamma T^{2} /\left(T+\gamma_{2}\right),
$$

where $E g(0), \gamma$, and $\gamma_{2}$ are known for a given semiconductor material (Refs. 13 and 29).

For a one-dimensional problem having an infinitely thick semiconductor, the rigorous relation is known,

$$
\begin{aligned}
E[\Psi(x)]= & \pm\{[\exp (-\beta \Psi)+\beta \Psi-1] \\
& \left.+[\exp (\beta \Psi)-\beta \Psi-1] n_{0} / p_{0}\right\}^{1 / 2} \sqrt{2} / \beta L_{D},
\end{aligned}
$$

with a positive sign for $\Psi>0$ and a negative sign for $\Psi \leqslant 0$, where $\Psi(x)$ is abbreviated as $\Psi .^{29}$ The extrinsic Debye length $L_{D}$ is $L_{D}=\left(\varepsilon_{s} / e p_{0} \beta\right)^{1 / 2}$.

Especially, Eq. (9) becomes at $x=0$,

$$
\begin{aligned}
E_{s}= & \pm\left\{\exp \left(-\beta \Psi_{s}\right)+\beta \Psi_{s}-1\right. \\
& \left.+\left[\exp \left(\beta \Psi_{s}\right)-\beta \Psi_{s}-1\right] n_{0} / p_{0}\right\}^{1 / 2} \sqrt{2} / \beta L_{D} .
\end{aligned}
$$

For $l_{f} \gg$ the thickness of space charge layer in the ferroelectric, from Eq. (8b), we have a similar relation in the ferroelectric,

$$
\begin{aligned}
E= & \pm\{\exp (-\beta \Phi)+\beta \Phi-1 \\
& \left.+[\exp (\beta \Phi)-\beta \Phi-1] n_{f_{0}} / p_{f_{0}}\right\}^{1 / 2} \sqrt{2} / \beta L_{D f},
\end{aligned}
$$

with a positive sign for $\Phi<0$ and a negative sign for $\Phi \geqslant 0$, where $p_{f_{0}}$ and $n_{f_{0}}$ are the hole carrier density and the electron destiny in the ferroelectric at $x=\infty$, and $\Phi(x)=\Psi(x)$ $-\Psi\left(x=-l_{f}-l_{d}\right)$ and $\Phi\left(-l_{d}\right) \equiv \Psi_{f}$. We assume $E=0$ at $x=-l_{f}-l_{d}$ and $x=\infty$ throughout this article. Consequently, the net charges in the space-charge layer in the semiconductor and the ferroelectric are $-D$ and $\varepsilon_{0} E_{f}$, respectively.

The $p_{0}$ and $n_{0}$ at $T$ and the extrinsic Debye length in ferroelectric $L_{D f}$ are given by

$$
\begin{gathered}
p_{f_{0}}=\left\{N_{A^{f}}-N_{D^{f}}+\left[\left(N_{A^{f}}-N_{D f}\right)^{2}+4 n_{i f}^{2}\right]^{1 / 2}\right\} / 2, \\
n_{f_{0}}=n_{i f}^{2} / p_{f_{0}}, \\
L_{D f}=\left(1 / e p_{0} \beta\right)^{1 / 2},
\end{gathered}
$$

where $n_{i f}$ is the intrinsic carrier density. The thickness of the space-charge layer in ferroelectrics for the accumulation and the inversion is estimated to be approximately 1 and $10 \mathrm{~nm}$, respectively, using the Boltzmann distribution. When $l_{f}$ is

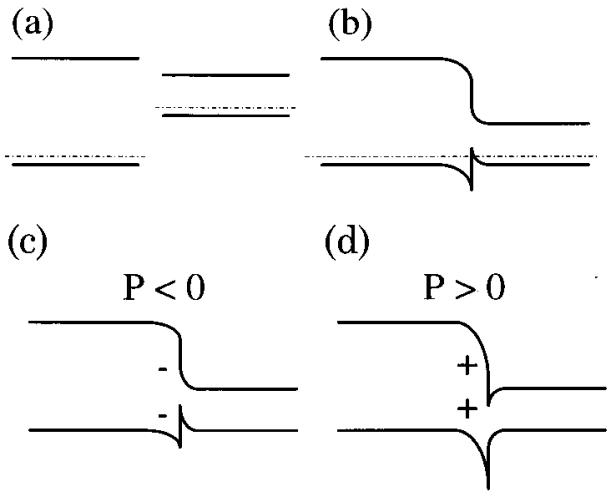

FIG. 2. Schematic diagram showing construction of a band diagram of a $p$-type-ferroelectric/p-type-semiconductor heterostructure. (a) before junction formation without ferroelectric $P_{s}$, (b) after junction formation without ferroelectric $P_{s}$. (c),(d) after junction formation with a negative and a positive $P_{s}$ 's.

below these thicknesses, the subsequent results are invalid. The maximum carrier density in the space-charge layer becomes unphysically large. This difficulty is overcome using the Fermi-Dirac distribution, which should slightly increase the thickness.

Equations (7), (9a), and (9b) define the charge distribution in a $\mathrm{F} / \mathrm{I} / \mathrm{S}$ as shown in Fig. 1. This scheme fits directly into the MOS diode theory. ${ }^{29}$ Equations (7), (9a), and (9b) also represent a semiconductor heterojunction, e.g., a $p p$ heterojunction or a $p n$ heterojunction. Indeed, well-known equations for the depletion layer in the metal/semiconductor contact can also be derived from Eqs. (9a) or (9b). Figure 2 is drawn in accordance with semiconductor heterojunction theory. ${ }^{30,31}$ By inserting on insulator to Fig. 2, Fig. 1 is obtained. The effect of the heterojunction on carrier distribution is expressed by $\delta \phi$. Using the notation in Fig. $1, \delta \phi$ is

$$
\delta \phi=E_{F s}-E_{F f}=\left[V_{s}-\delta_{s}-\left(V_{f}-\delta_{f}\right)\right],
$$

where $E_{F s}$ and $E_{F f}$ are the Fermi levels before formation of the junction.

For $\nabla P \neq 0$, the dielectric constant of the ferroelectric $\varepsilon_{f}$ effectively larger than unity can be defined as $\varepsilon_{0} \varepsilon_{f}$ $=\partial D / \partial E$.

This modifies Eq. (8b) and, therefore, $L_{D f}$ in Eq. (9b),

$$
\begin{gathered}
\Delta \Psi(x)=e\left(N_{D f}^{+}-N_{A f}^{-}+p_{f}-n_{f}\right) / \varepsilon_{0} \varepsilon_{f}, \\
L_{D f}=\left(\varepsilon_{f} / e p_{0} \beta\right)^{1 / 2} .
\end{gathered}
$$

In this case, we modify Eq. (6b) as,

$$
D=\varepsilon_{f} \varepsilon_{0} E_{f}+P,
$$

where $\varepsilon_{f}$ is the linear dielectric constant.

Using Eq. (9a), Eq. (4) is rewritten as

$$
F_{s}=\varepsilon_{s} \varepsilon_{0} \int_{0}^{\Psi_{s}} d \Psi E(\Psi) / 2
$$

Similarly, using Eq. (7) and Fig. 1, the second to last term in Eq. (2) is written as 


$$
\begin{aligned}
& -\varepsilon_{f} \varepsilon_{0} \int_{\Psi\left(-l_{f}-l_{d}\right)}^{\Psi\left(-l_{d}\right)} d \Psi E(\Phi) / 2 \\
& =-\varepsilon_{f} \varepsilon_{0} \int_{\delta \phi+V_{e}}^{\delta \phi+V_{e}+\Psi_{f}} d \Psi E\left(\Psi-\delta \phi-V_{e}\right) / 2 \\
& =-\varepsilon_{f} \varepsilon_{0} \int_{0}^{\Psi_{f}} d \Psi E(\Psi) / 2
\end{aligned}
$$

where, $E(\Psi)$ is given by Eq. (9b). Finally, Eq. (1) for $\nabla P$ $=0$ is reduced to

$$
\begin{aligned}
F= & F_{0}+\varepsilon_{f} \varepsilon_{0} / 2 l_{f} \int_{-l_{f}-l_{d}}^{-l_{d}} d x E_{f}^{2}+\varepsilon_{s} \varepsilon_{0} / 2 l_{f} \int_{0}^{\infty} d x E(x)^{2} \\
& +D^{2} l_{e} / 2 l_{f} \varepsilon_{0}-P\left(\delta \phi+V_{e}\right) / l_{f} \\
= & F_{0}-\varepsilon_{f} \varepsilon_{0} / 2 l_{f} \int_{0}^{\Psi_{f}} d \Psi E+\varepsilon_{s} \varepsilon_{0} / 2 l_{f} \int_{0}^{\Psi_{s}} d \Psi E \\
& +D^{2} l_{e} / 2 l_{f} \varepsilon_{0}-P\left(\delta \phi+V_{e}\right) / l_{f} .
\end{aligned}
$$

Minimization of Eq. (11) gives an equilibrium $D$ under the conditions of Eqs. (6a), (6b), (7), (9), (9a), and (9b) for given $l_{e}, l_{f}, \delta \phi, V_{e}, T$, semiconductor material parameters, and GLD parameters.

The effect of $\nabla P \neq 0$ is approximately treated by postulating $\varepsilon_{f}>1$. The following numerical results show that this modifies the result only slightly.

\section{B. Approximate analytical solution}

Equation (11a) is approximately estimated for $\delta \phi+V_{e}$ $=0$ using a simplified equation for the electric-field distribution, when both ferroelectric and the semiconductor satisfy the strong inversion or the accumulation condition

$$
E(x) \approx D / \varepsilon_{s} \varepsilon_{0}(1+x / 2 d),
$$

where $d \approx \varepsilon_{s} \varepsilon_{0} / D \beta .^{32}$ Using this equation, we have

$$
\begin{aligned}
F & \approx F_{0}+\beta^{-1}|D-P| / l_{f}+\beta^{-1}|D| / l_{f}+D^{2} l_{e} / 2 l_{f} \varepsilon_{0} \\
& =F_{0}+\beta^{-1}|P| / l_{f}+D^{2} l_{e} / 2 l_{f} \varepsilon_{0} .
\end{aligned}
$$

The first, second, third, and last terms represent the bulk free energy, the depolarization, the semiconductor charging, and the insulator polarization energies, respectively. We can approximate $P \approx P_{0}$ in Eq. (11) except for the proximity of the phase transition, where $P_{0}$ is $P_{s}$ of the bulk ferroelectric.

The effect of the $c$-axis $\left(P_{s}\right)$ orientation $(\varphi)$ can be included by changing $|P|$ to $|P \cos \varphi|$ in Eq. (12). The effect of $\varphi \neq 0$ is much smaller than that in an insulating ferroelectric.

Equation (12) is not precise enough to estimate $D$ to minimize $F$. Using Eqs. (6a) and $\left(6 \mathrm{~b}^{\prime}\right)$, and differentiating $F$ in Eq. (11b) by $D, D$ for minimizing $F$ is given by

$$
\begin{aligned}
d F / d D \approx & D l_{e} / l_{f} \varepsilon_{0}+\left(d \Psi_{f} / d D\right)\left(P_{0}-D\right) / l_{f} \\
& +\left(d \Psi_{s} / d D\right) D / l_{f}=0 .
\end{aligned}
$$

We use approximate forms of $\Psi_{s}$ and $\Psi_{f}$ to solve this equation for the following conditions of the ferroelectric and the semiconductor: inversion, weak inversion and depletion, weak depletion and weak accumulation, and accumulation. The expressions of $\Psi_{s}$ and $\Psi_{f}$ for these conditions are given in Appendix D.

We consider a $p$-type (hole-type) ferroelectric without losing generality. In the following, we introduce two quantities,

$$
\begin{gathered}
\mathcal{D}=\varepsilon_{0} \beta^{-1} / L_{D f} \\
\boldsymbol{D}=\varepsilon_{0} \beta^{-1} / l_{e},
\end{gathered}
$$

where both have a form of an electric flux due to a thermal potential. Furthermore, we define the critical $l_{e}\left(l_{e}^{\text {crit }}\right)$. The effect of the insulating layer is dominant for $l_{e}$ larger than $l_{e}^{\text {crit }} . l_{e}^{\text {crit }}$ is given by equating $P_{0}^{2} l_{e} / 2 \varepsilon_{0} l_{f}$ with $\left|F_{0}\left(P_{0}\right)\right|$ $\approx\left|\left\{T-\left[\theta-C F_{s 1}(\underline{S})\right]\right\} / 2 \varepsilon_{0} C P_{0}^{2}\right|$ using Eq. (2b), and this gives $l_{e}^{\text {crit }} \approx l_{f}\left[\theta-C F_{s 1}(\underline{S})\right] / C$ at $25^{\circ} \mathrm{C}=R T$.

First, we solve Eq. (13) for a $p$-type semiconductor.

(a) Ferroelectric in weak accumulation and semiconductor in accumulation.

This condition should be satisfied for $l_{e}<l_{e}^{\text {crit }}$ with $\delta \phi \approx 0$. Equation (13) is reduced to

$$
D l_{e} / l_{f} \varepsilon_{0}-\left(P_{0}-D\right) \beta^{-1} / \mathcal{D} l_{f}+2 \beta^{-1} / l_{f}=0 .
$$

This gives

$$
D=P_{0}\left(1-2 \mathcal{D} / P_{0}\right) L_{D f} /\left(l_{e}+L_{D f}\right) \approx P_{0} L_{D f} /\left(l_{e}+L_{D f}\right) .
$$

(b) Ferroelectric in weak depletion and semiconductor in accumulation, ferroelectric in depletion or weak inversion and semiconductor in accumulation, ferroelectric in weak inversion or depletion and semiconductor in inversion.

The first two conditions should be satisfied for $l_{e}<l_{e}^{\text {crit }}$ with $\delta \phi<0$, and the last is for $l_{e}<l_{e}^{\text {crit }}$. The solution for the first condition is the same as Eq. (16a). For the second and the third cases, we have

$$
D l_{e} / l_{f} \varepsilon_{0}-\left(P_{0}-D\right)^{2} \beta^{-1} / \mathcal{D}^{2} l_{f}+2 \beta^{-1} / l_{f}=0 .
$$

This gives

$$
\begin{aligned}
D & =P_{0}+\mathcal{D}^{2} / 2 \boldsymbol{D}-\left(\mathcal{D} P_{0} l_{e} / L_{D f}+\mathcal{D}^{4} / 2 \boldsymbol{D}^{2}\right)^{1 / 2} \\
& \approx P_{0}-\left(\mathcal{D} P_{0} l_{e} / L_{D f}\right)^{1 / 2} .
\end{aligned}
$$

(c) Ferroelectric in accumulation and semiconductor in weak accumulation, ferroelectric in inversion and semiconductor in weak depletion.

This condition should be satisfied for $l_{e}>l_{e}^{\text {crit }}$.

$$
D l_{e} / l_{f} \varepsilon_{0}-2 \beta^{-1} / l_{f}+D \beta^{-1} / \mathcal{D} l_{f}=0 .
$$

This gives

$$
D=2 \mathcal{D} /\left(1+l_{e} / L_{D f}\right)=2 \varepsilon_{0} \beta^{-1} /\left(l_{e}+L_{D f}\right) .
$$

(d) Ferroelectric in inversion and semiconductor in weak inversion or depletion.

This condition should be satisfied for $l_{e}>l_{e}^{\text {crit }}$.

$$
D l_{e} / l_{f} \varepsilon_{0}-2 \beta^{-1} / l_{f}+D^{2} \beta^{-1} / \mathcal{D}^{2} l_{f}=0 .
$$

This gives 


$$
D=\mathcal{D}\left[\left(2+\mathcal{D}^{2} / 4 \boldsymbol{D}^{2}\right)^{1 / 2}-\mathcal{D} / 2 \boldsymbol{D}\right] .
$$

The value of $l_{e}^{\text {crit }}$ is less than 1-2 nm for $100 \mathrm{~nm}$-thick $\mathrm{BaTiO}_{3}$ and $\mathrm{PbTiO}_{3}$ thin films. Therefore, Eqs. (16c) and (16d) describe the electric flux in most of the F/I/S.

The solutions for both ferroelectric and semiconductor in accumulation and ferroelectric in inversion and semiconductor inversion are approximated by Eqs. (16a)-(16d). When the semiconductor is $n$-(electron carrier) type, the depletion, the weak inversion, and the accumulation for the semiconductor need to be examined. The solutions for these conditions are also given by Eqs. (16a), (16c), and (16d).

We should note that $D$ is independent of $l_{f}$ and the ferroelectric GLD parameters for a finite $l_{e}$ [Eqs. (16c) and (16d)] and that $D$ is inversely proportional to $l_{e}$. Contrarily, the minimum $l_{f}, l_{f}^{\mathrm{min}}$, at which $P_{s}$ exists is determined by the GLD parameters.

The minimum $F, F_{\min }$, is obtained by substituting $D$ in the above four cases into Eq. (12), and $l_{f \text { min }}$ is given by $F_{\text {min }}=0$. Because $D$ is independent of $l_{f}$, using Eq. (12), we have

$$
l_{f}^{\min }=\left(\beta^{-1}|P|+D^{2} l_{e} / 2 \varepsilon_{0}\right) /\left|F_{0}\left(P_{0}\right)\right| .
$$

Equation (17) becomes

$$
\begin{aligned}
& l_{f}^{\min }=\beta^{-l}|P| /\left|F_{0}\left(P_{0}\right)\right| \text { for } l_{e}<l_{e}^{\text {crit }}, \\
l_{f}^{\min }= & \beta^{-1}\left[|P|+2 \varepsilon_{0} \beta^{-1} l_{e} /\left(l_{e}+L_{D f}\right)^{2}\right] /\left|F_{0}\left(P_{0}\right)\right| \\
\approx & \beta^{-1}|P| /\left|F_{0}\left(P_{0}\right)\right| \text { for } l_{e}>l_{e}^{\text {crit }} .
\end{aligned}
$$

The functional form indicates that a low $P_{s}$ and a low thermodynamic linear susceptibility suppresses the depolarization instability.

We estimate now the stability of $P_{s}$ for typical material parameters. Typical values of $F_{0}$ for $\mathrm{BaTiO}_{3}$ and $\mathrm{PbTiO}_{3}$ are $1 \mathrm{~J} / \mathrm{cm}^{3}$ and $70 \mathrm{~J} / \mathrm{cm}^{3}$ at $300 \mathrm{~K}$, respectively, and their $P_{s}$ 's are $25 \mu \mathrm{C} / \mathrm{cm}^{2}$ and $75 \mu \mathrm{C} / \mathrm{cm}^{2}$, respectively. $\beta^{-1}$ is $0.026 \mathrm{~V}$ at $300 \mathrm{~K} . N_{D f}$ and $N_{A f}$ in ferroelectric films are typically 0 and $10^{18} \mathrm{~cm}^{-3} .{ }^{33}$ Therefore, we have $\mathcal{D} \sim 0.02 \mu \mathrm{C} \mathrm{cm}^{-2}$ and $L_{D f} \sim 10^{-7} \mathrm{~cm}$.

In F/S $\left(l_{e}=0\right), D$ is close to $P_{s}$ of the bulk, as indicated by Eqs. (16a) and (16b). The stability of $P_{s}$ is dependent on doping in ferroelectric as seen in the $p_{0}$ dependence of $\mathcal{D}$ $\left(\propto \sqrt{p_{0}}\right)$. In F/I/S $\left(l_{e} \neq 0\right), D$ is a small fraction of $P_{s}$ of the bulk for the accumulation in ferroelectric. Their typical value is $0.1 \mu \mathrm{C} \mathrm{cm}^{-2}\left(1 \mathrm{~nm} / l_{d}\right)\left(\varepsilon_{d} / 3.9\right)$. The degree of stability is evaluated by $l_{f}^{\mathrm{min}} ; 0.28 \mathrm{~nm}$ for $\mathrm{PbTiO}_{3}, 1 \mathrm{~nm}$ for $\mathrm{KNbO}_{3}$, and $7 \mathrm{~nm}$ for $\mathrm{BaTiO}_{3}$. The $l_{f}^{\text {min }}$ has no physical meaning below $1 \mathrm{~nm}$ because it is shorter than the space-charge layer thickness.

When ferroelectric is sandwiched by two insulator/ semiconductor multilayers, the depolarization energy should be given by doubling the above values. The limit of $l_{e}=\infty$ in this structure corresponds to an insulator/ferroelectric/ insulator structure, e.g., a ferroelectric particulate in vacuum. In this case $D$ is zero, but a unidomain ferroelectric phase can exist as long as $l_{f}$ is thicker than $l_{f}^{\text {min }}$ even if there is no disorder or surface states.

\section{NUMERICAL RESULTS}

Firstly, Eq. (11) is solved numerically for a $c$-axis oriented unidomain $\mathrm{PbTiO}_{3}$ /insulator/Si with $N_{D}=N_{D f}=0$, where the insulator is $\mathrm{SiO}_{2}$ having $\varepsilon_{d}=3.9$, i.e., $l_{e}=l_{d} / 3.9$ and $\mathrm{Si}$ is a $p$ type. In the following, the external bias $V_{e}$ is set as zero. $\mathrm{Si}$ is a standard semiconductor. The Pb-related ferroelectric is frequently used in experiments of this sort, and $\mathrm{PbTiO}_{3}$ has a good resistance to depolarization instability as shown in Ref. 13. As discussed in the previous analytical examination and as shown later, the choice of the GLD parameters, i.e., the ferroelectric material is not important as those of the semiconductor and the ferroelectric as a semiconductor. The GLD parameters in Eq. (11) and the semiconductor material parameters are listed in Refs. 13, 26, and 29. We used $3.2 \mathrm{eV}$ for the band gap of the ferroelectric $E g f$ at $25^{\circ} \mathrm{C},{ }^{33}$ and the approximate intrinsic carrier density $n_{i f}$ at $25^{\circ} \mathrm{C}$ was estimated using

$$
n_{i f}=n_{i} \exp [-\beta(E g f-E g) / 2]
$$

where $E g$ and $n_{i}$ are the intrinsic carrier density and the band gap of the semiconductor at RT of which these values are known, respectively. By using $E g$ and $n_{i}$ of $\mathrm{Si}$, we tentatively estimated $n_{i}$ of $\mathrm{Ge}$ and GaAs, which were close to the experimental values.

The band offset $\delta \phi$ is estimated as $-1.7 \mathrm{eV}$ using Eq. (7b), when $\mathrm{PbTiO}_{3}$ has a high acceptor density $N_{A f}$ that is usually considered to be created by oxygen vacancy, and its Fermi level is close to the top of the valence band. Here, $V_{f}$ and $V_{s}$ are 7 and $5.3 \mathrm{~V}$, respectively, and $\delta_{f}-\delta_{s}$ is approximated as zero.

In experiments, the domain motion is pinned by various defects in the ferroelectric films and at the interfaces. Therefore, a complete domain switching cannot be expected. Therefore, the absolute value of $D$ experimentally estimated should be smaller than that estimated below, by a factor of 2 to 3 in some cases.

Figure 3 shows typical results for the free energy $F$ vs the electric flux $D$ [Figs. 3(a)-3(c)] and $F$ vs the semiconductor surface potential $\Psi_{s}$ [Figs. $\left.3(\mathrm{~d})-3(\mathrm{f})\right]$. As $l_{d}$ increases, the absolute value of $D$ as well as the width of the $F$ minimum drastically decreases. Nevertheless, the lines have welldefined double minima and shift parallel as $N_{A}$ increases. Namely, the unidomain ferroelectric phase is bistable even for a relatively large $l_{d}$, in contrast with the result for insulating ferroelectrics. ${ }^{13}$ When $\delta \phi=0$ and $N_{A}=10^{10}$ and $10^{14} \mathrm{~cm}^{-3}, D$ becomes a small fraction of $P_{s}$ of the bulk even for $l_{d}=0$. The change of $F-\Psi_{s}$ curves with $l_{d}$ is moderate as compared with that of $F-D$ curves. Figure 4 shows the ferroelectric surface potential $\Psi_{f}$ vs $D$ and $\Psi_{s}$ vs $D$ for $\delta \phi=-1.7 \mathrm{~V}$ corresponding to Figs. 3(a)-3(c). The values of $\Psi_{f}$ at the equilibrium are read by referring to Figs. 3(a)-3(c) and are only weakly dependent on $l_{d}$, reaching $-1 \mathrm{~V}$ for accumulation and exceeding $3 \mathrm{~V}$ for inversion.

Figure 5 demonstrates that there is little $l_{f}$ dependence of $D$ and that ferroelectric phase can exist for a very short $l_{f}$ as long as the space-charge layer formation is allowed. [For a very short $l_{f}$ Eq. (9b) needs to be replaced by numerical $E-\Phi$ relation. This increases $l_{f}^{\mathrm{min}}$ slightly and makes $D$ more dependent on $l_{f}$.]

Figures 6-8 show the temperature dependence of $D, F$, and $P_{s}$. In Figs. 6 and $7, \delta \phi$ is 0 and $\varepsilon_{f}$ is 1 , while the thin and the thick lines in Fig. 8 correspond to $\varepsilon_{f}=1$ and $\varepsilon_{f}$ $=100$ with $\delta \phi=-1.7 \mathrm{~V}$, respectively. The choice of $\varepsilon_{f}>1$ 

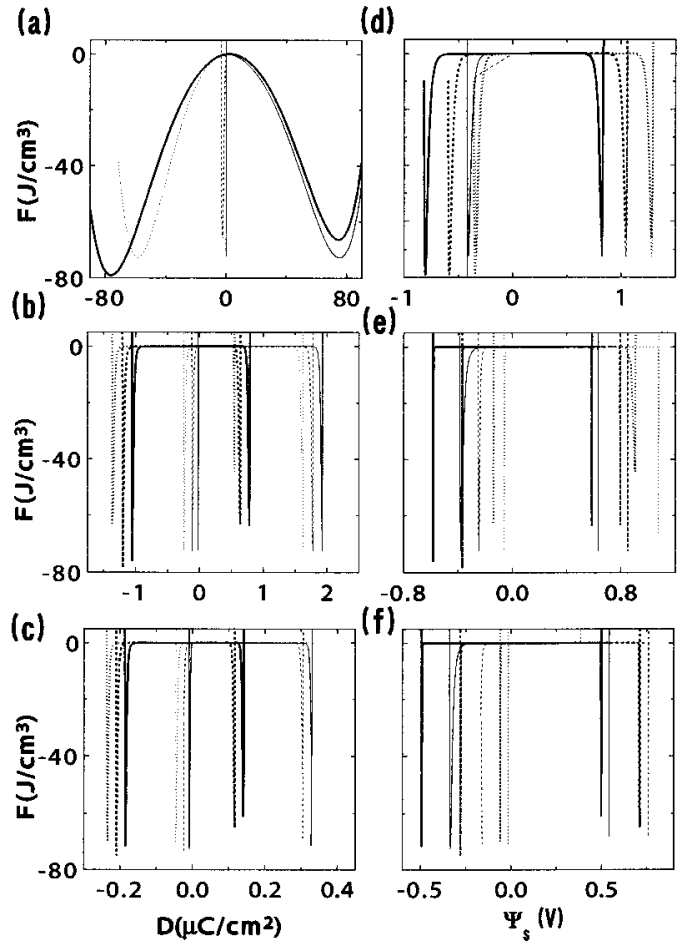

FIG. 3. Total free energy $F$ to induce $D$ and the semiconductor surface potential $\Psi_{s}$ for $\varepsilon_{f}=1, N_{A f}=10^{18}$, and $l_{f}=200 \mathrm{~nm}$ in $\mathrm{PbTiO}_{3} / \mathrm{SiO}_{2} / \mathrm{Si}$ at $25^{\circ} \mathrm{C}$. (a)-(c) show $F$ vs $D$ for $l_{d}=0,5$, and 30 $\mathrm{nm}$, respectively, and (d)-(f) show $F$ vs $\Psi_{s}$ for $l_{d}=0,5$, and $30 \mathrm{~nm}$, respectively. The thin and thick lines are for $\delta \phi=0$ and $-1.7 \mathrm{~V}$, respectively. The lines $(-),(--)$, and $(\cdots)$ correspond to $N_{A}$ $=10^{10}, 10^{14}$, and $10^{18} \mathrm{~cm}^{-3}$, respectively.

represents the effect of $\nabla P \neq 0$ as discussed in Sec. II A. $D$ near RT is insensitive to $\varepsilon_{f}$ and mainly determined by $l_{d}$ and partly by $N_{A}$ and $\delta \phi$ (Fig. 8). Furthermore, for $\delta \phi=-1.7 \mathrm{~V}$ and $D<0$, e.g., the accumulation condition, $D$ is almost independent of $N_{A}$ and $\varepsilon_{f}$. On the other hand, $D$ at higher temperature is reduced as $\varepsilon_{f}$ increases for $\delta \phi=-1.7 \mathrm{~V}$ and $D>0$. The effect of $\delta \phi$ is significant at short $l_{f}$ or near $T$ $\sim T_{c}$. The increase of $D$ with the temperature indicated by the lines for $N_{A}=10^{10} \mathrm{~cm}^{-3}$ in Fig. 6 is due to the reduction of the semiconductor band gap. In both Figs. 6 and 8, $T_{c}$ is weakly dependent on $l_{f}$ but almost independent of $l_{d}$ and $N_{A}$.

The temperature dependence and the value of $F$ and $P$ are mainly determined by $l_{f}$. Their values are close to the bulk value at a relatively large thickness of $200 \mathrm{~nm}$ in agreement with the analytical results in Sec. III. However, the size effect is manifested in the phase transition that becomes sharpened as $l_{f}$ shortens, as seen from the comparison of the lines for $l_{f}=200$ and $5 \mathrm{~nm}$. The sharpening of the phase transition is similar to the results for insulating ferroelectric semiconductor structure (Ref. 13).

This is more evidently seen in the derivatives of $P$ by the temperature in Fig. 9, which corresponds to the pyroelectric current. For $l_{f}=5 \mathrm{~nm}$ with $l_{d}=0$, large peaks of $d P / d T$ and $d D / d T$ are found at the temperature lower than the bulk $T_{c}$. For $l_{f}=200 \mathrm{~nm}$ with $l_{d}=5 \mathrm{~nm}$, the peak height of $d D / d T$ is reduced proportionally to $D$ in Fig. 6 .

Dependence of $D$ on $\delta \phi$ is shown in Fig. 10. It should be reminded that for a $p$-type $\mathrm{PbTiO}_{3}$ /insulator/ $p$-type $\mathrm{Si}, \delta \phi$ is

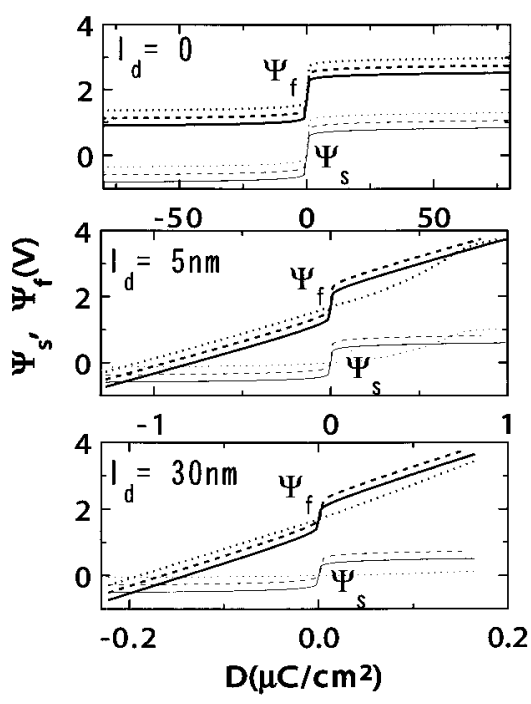

FIG. 4. $\Psi_{s}$ and $\Psi_{f}$ to induce $D$, corresponding to Figs. 1(a)1(f), and Figs. 2(a) -2 (c) are for $l_{d}=0,5$, and $30 \mathrm{~nm}$ with $\delta \phi=-1.7$ $\mathrm{V}$, respectively. The thin and the thick lines show $\Psi_{s}$ and $\Psi_{f}\left(\varepsilon_{f}\right.$ $=1, N_{A f}=10^{18} \mathrm{~cm}^{-3}, T=25^{\circ} \mathrm{C}$. The lines (-), (---), and ( $\left.\cdots\right)$ correspond to $N_{A}=10^{10}, 10^{14}$, and $10^{18} \mathrm{~cm}^{-3}$, respectively.

estimated to be $-1.7 \mathrm{~V}$. For $l_{f}>100 \mathrm{~nm}$, the bistability of the ferroelectric phase is relatively insensitive to the choice of $\delta \phi$, although the value of $D$ is changed. Because $\delta \phi$ is about $-2.7 \mathrm{~V}$ for an $n$-type $\mathrm{Si}$, the bistability is also expected when the semiconductor is $n$-type $\mathrm{Si}$. In Fig. 11, the doping dependence of $D$ is examined. Marked doping dependence of a negative $D$ is seen for $\delta \phi=0$, but the doping dependence of a negative and a positive $D$ 's are weak for $\delta \phi=-1.7 \mathrm{~V}$. Nonetheless, the doping and $\delta \phi$ dependence is evident as compared with the results for an insulating ferroelectric model (Ref. 13). Namely, $D$ is sensitive to the semiconductor parameters in a semiconducting ferroelectric model.

The preceding results indicated that $l_{d}$ is the main factor determining $D$ in $\mathrm{F} / \mathrm{I} / \mathrm{S}$, when the band gap of the semiconductor is sufficiently smaller than the ferroelectric band gap. Figures 12-14 show $l_{d}$ dependence of $D$. The GLD parameters for Fig. 12 are those of $\mathrm{PbTiO}_{3}$ as in Figs. 3-11, while the GLD parameters for Fig. 13 are those of $\mathrm{BaTiO}_{3}$. The rest of the parameters used are same for both Figs. 12 and 13. The thick lines in Fig. 12 and the lines in Fig. 13 are for $N_{A f}=10^{18} \mathrm{~cm}^{-3}$. Each line of Fig. 13 is very close to the corresponding one of Fig. 12 having the same $N_{A}$ and $\delta \phi$ at nonzero $l_{d}$. This demonstrates that $D$ at nonzero $l_{d}$ is insensitive to the choice of the GLD parameters, in agreement with the analytical results in Sec. II B.

The thin lines in Fig. 12 are for $N_{A f}=10^{10} \mathrm{~cm}^{-3}$. The effect of lowering $N_{A f}$ is similar to that of reducing $\delta \phi$ or a increasing $N_{A}$. For $\delta \phi=0$, the absolute $D$ values $(D<0)$ for $N_{A f}=10^{10} \mathrm{~cm}^{-3}$ are much higher than those for $N_{A f}$ $=10^{18} \mathrm{~cm}^{-3}$. Except the proximity of $l_{d}=0, D$ is inversely proportional to $l_{d}$ (Fig. 14), which agrees with the analytical estimation in Sec. II B. The value of $D$ is of an order $0.1-1 \mu \mathrm{C} \mathrm{cm}^{-2}$ for $l_{d}=5-30 \mathrm{~nm}$, which is also consistent with the analytical result. In several experimental studies, ${ }^{11,12}$ $D$ is estimated to be a few $0.1 \mu \mathrm{C} \mathrm{cm}^{-2}$ for $l_{d}=5-10 \mathrm{~nm}$ 

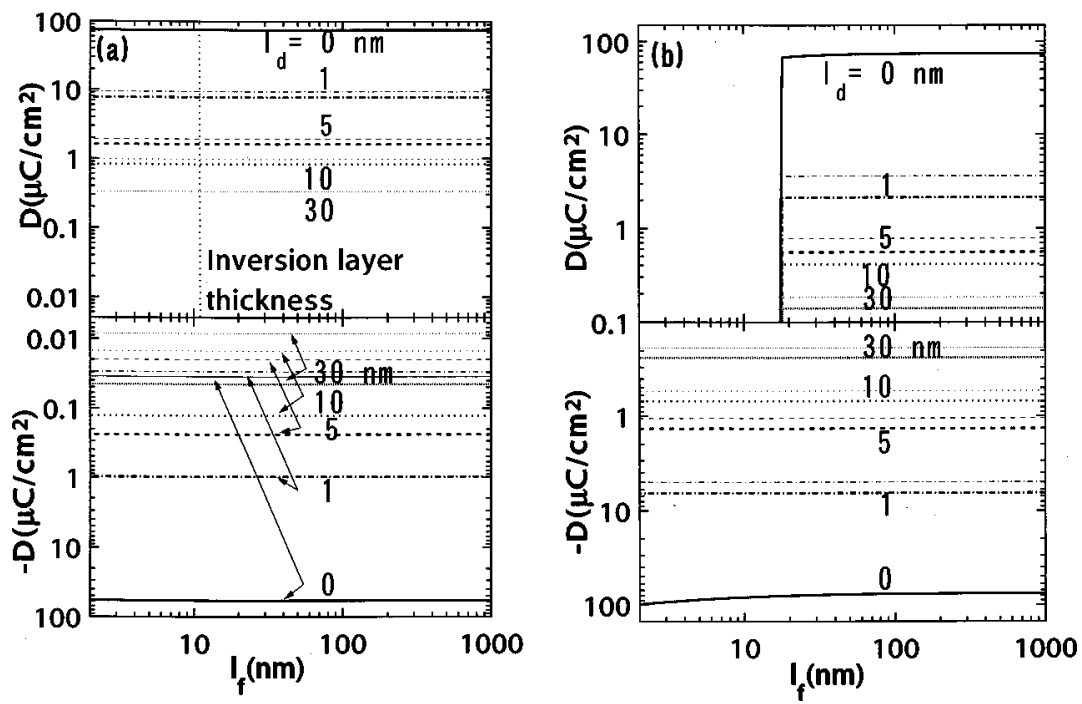

FIG. 5. Ferroelectric thickness $\left(l_{f}\right)$ dependence of $D$ for $\varepsilon_{f}=1$ and $N_{A f}=10^{18} \mathrm{~cm}^{-3}$, and $\delta \phi=0(\mathrm{a})$ and $-1.7 \mathrm{~V}(\mathrm{~b}) \mathrm{in} \mathrm{PbTiO}_{3} / \mathrm{SiO} \mathrm{S}_{2} / \mathrm{Si}$ at $25{ }^{\circ} \mathrm{C}$. The thin and the thick lines corresponds to $N_{A}=10^{10}$ and $10^{18} \mathrm{~cm}^{-3}$, respectively. The lines $(-),(-\cdot \cdot),(--),(\cdots)$, and $(\cdots \cdots)$ correspond to $l_{d}=0,1,5,10$, and $30 \mathrm{~nm}$, respectively. The space-charge layer in the ferroelectric is thick for $D>0$ (inversion) as indicated by the vertical lines. For $l_{f}<$ the space-charge layer thickness $D$ should vanish.

and agrees well with the present results. Furthermore, $D$ should be of order $0.1 \mu \mathrm{C} \mathrm{cm}^{-2}$ in $\mathrm{Bi}_{4} \mathrm{Ti}_{3} \mathrm{O}_{12} / 30 \mathrm{~nm}-\mathrm{SiO}_{2} / \mathrm{Si}$ by Sugibuchi. ${ }^{10}$

Secondly, Eq. (11) is solved for a $c$-axis oriented unidomain $\mathrm{BaTiO}_{3} / \mathrm{SrTiO}_{3}$ with $N_{A}=0$ and $l_{f}=10 \mathrm{~nm}$, where $\mathrm{SrTiO}_{3}$ is an $n$-(electron carrier) type. We have chosen $\mathrm{BaTiO}_{3}$ because it should be the most unstable among the well-known oxide ferroelectrics as analytically estimated in Sec. II B. Figure 15 shows the results for the $p$ - and the $n$-type $\mathrm{BaTiO}_{3}$, because the carrier type of the $\mathrm{BaTiO}_{3}$ film was not determined. In this calculation, we assumed the polarization at the surface exposed to the air is compensated by

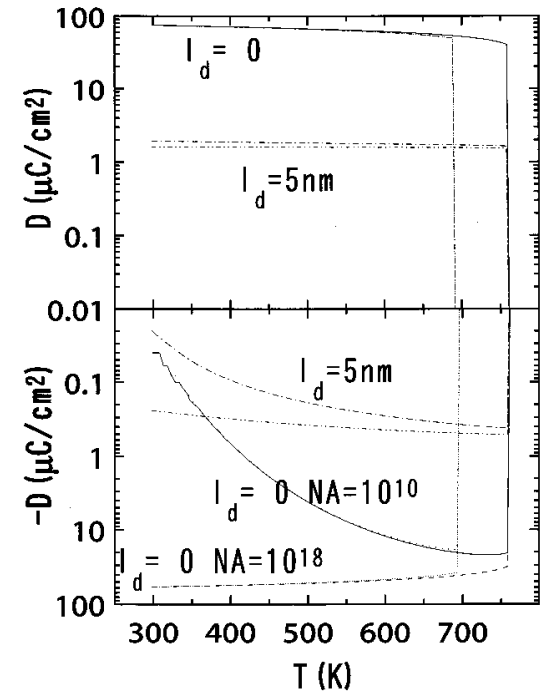

FIG. 6. Temperature dependence of $D$ for $\varepsilon_{f}=1$ and $N_{A f}$ $=10^{18} \mathrm{~cm}^{-3}$, and $\delta \phi=0$ in $\mathrm{PbTiO}_{3} / \mathrm{SiO}_{2} / \mathrm{Si}$. The three sets of lines $(-)$ and $(---),(\ldots)$ and $(\ldots \ldots)$, and $(-\cdots)$ and $(\ldots \ldots)$ correspond to $N_{A}=10^{10}$ and $10^{18} \mathrm{~cm}^{-3}$ for $l_{f}=200 \mathrm{~nm}$ and $l_{d}=0, N_{A}$ $=10^{10}$ and $10^{18} \mathrm{~cm}^{-3}$ for $l_{f}=5 \mathrm{~nm}$ and $l_{d}=0, N_{A}=10^{10}$ and $10^{18} \mathrm{~cm}^{-3}$ for $l_{f}=200 \mathrm{~nm}$ and $l_{d}=5 \mathrm{~nm}$. the contamination at the surface. The surface of $\mathrm{SrTiO}_{3}$ is reported to be oxygen deficient and becomes a $n$-type semiinsulator. For $p$ - and $n$-type $\mathrm{BaTiO}_{3}, \delta \phi$ is estimated to be approximately $-3 \mathrm{~V}$ and 0 , respectively. Therefore, the lines with $\delta \phi=-4,-3$, and $-2 \mathrm{~V}$ are plotted for the $p$-type, and the lines with $-1 \mathrm{~V}, 0$, and $-1 \mathrm{~V}$ are plotted for the $n$-type. The ferroelectric phase of $\mathrm{BaTiO}_{3}$ is stable, at least, at one polarity with its $D$ close to the bulk value. This would explain the ferroelectric phase of 14 nm-thick $\mathrm{BaTiO}_{3}$ detected by $\mathrm{x}$-ray diffraction. However, the bistability is expected
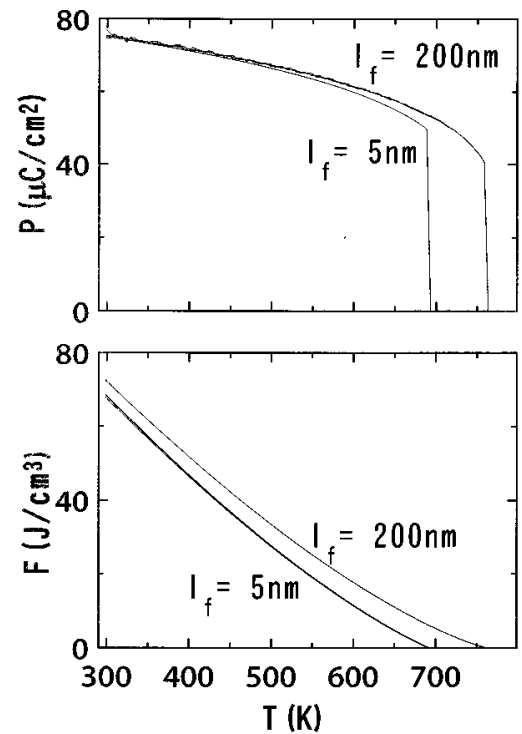

FIG. 7. Temperature dependence of the spontaneous polarization (a) and the total free energy $F$ (b) for $\varepsilon_{f}=1$ and $N_{A f}$ $=10^{18} \mathrm{~cm}^{-3}$, and $\delta \phi=0, \quad N_{A}=10^{10}$ and $10^{18} \mathrm{~cm}^{-3}$ in $\mathrm{PbTiO}_{3} / \mathrm{SiO}_{2} / \mathrm{Si}$, corresponding to Fig. 6. (a) shows only positive $P$, while (b) shows $F$ for a positive and a negative $D$. The lines denoted as $l_{f}=200 \mathrm{~nm}$ and $5 \mathrm{~nm}$ are for $l_{f}=200 \mathrm{~nm}$ with $l_{d}=0$ and $5 \mathrm{~nm}$, and $l_{f}=5 \mathrm{~nm}$ with $l_{d}=0$, respectively. 


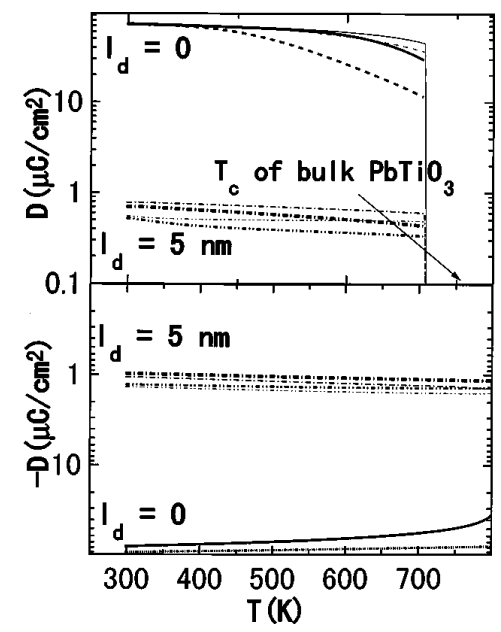

FIG. 8. Temperature dependence of $D$ for $N_{A f}=10^{18} \mathrm{~cm}^{-3}$ and $\delta \phi=-1.7 \mathrm{~V}$ in $\mathrm{PbTiO}_{3} / \mathrm{SiO}_{2} / \mathrm{Si}$ at $25^{\circ} \mathrm{C}$. The thin and the thick lines corresponds to $\varepsilon_{f}=1$ and 100 . The three sets of lines (-) and $(--),(\cdots)$ and $(\cdots \cdots)$, and $(-\cdot-\cdot)$ and $(-\cdots \cdots)$ correspond to the $N_{A}, l_{f}$, and $l_{d}$ values in caption of Fig. 6, respectively. No stable $D>0$ exists for $l_{f}=5 \mathrm{~nm}$.

only for a $n$-type $\mathrm{BaTiO}_{3}$ with $\delta \phi \approx 0 \mathrm{~V}$, which may not always be realized in real films. The loss of bistability should not be much changed, even if the donor concentration is reduce to $10^{10} \mathrm{~cm}^{-3}$ from $10^{16} \mathrm{~cm}^{-3}$ used in Fig. 15. However, $\mathrm{BaTiO}_{3}$ becomes bistable by increasing its thickness or changing $\mathrm{SrTiO}_{3}$ to a semiconductor having a narrower band gap. The latter is already demonstrated in Fig. 13.

\section{DISCUSSION: THE MULTIDOMAIN EFFECT, THE STABILITY OF SPACE CHARGE LAYER IN THE SEMICONDUCTOR, THE ABSORPTION CURRENT AND THE SURFACE LAYER}

(i) Stability of a multidomain ferroelectric phase in a ferroelectric/insulator.

Multidomain formation is a common mechanism to reduce the electrostatic energy in ferroelectrics without electrodes. In the preceding section, we have not included this

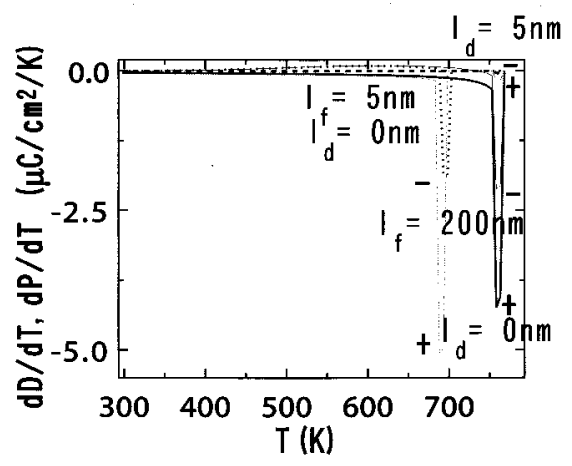

FIG. 9. Temperature derivatives of $D$ and $P$ that are shown in Figs. 6 and 7 for $\varepsilon_{f}=1$ and $N_{A f}=10^{18} \mathrm{~cm}^{-3}$, and $\delta \phi=0$ in $\mathrm{PbTiO}_{3} / \mathrm{SiO}_{2} / \mathrm{Si}$. There are three combinations of $l_{f}$ and $l_{d}$ and are indicated by labels, i.e., $l_{f}=200 \mathrm{~nm}$ and $l_{d}=0, l_{f}=5 \mathrm{~nm}$ and $l_{d}$ $=0, l_{f}=5 \mathrm{~nm}$ and $l_{d}=0$. The symbols + and - indicate the derivative of a positive and a negative $P$.

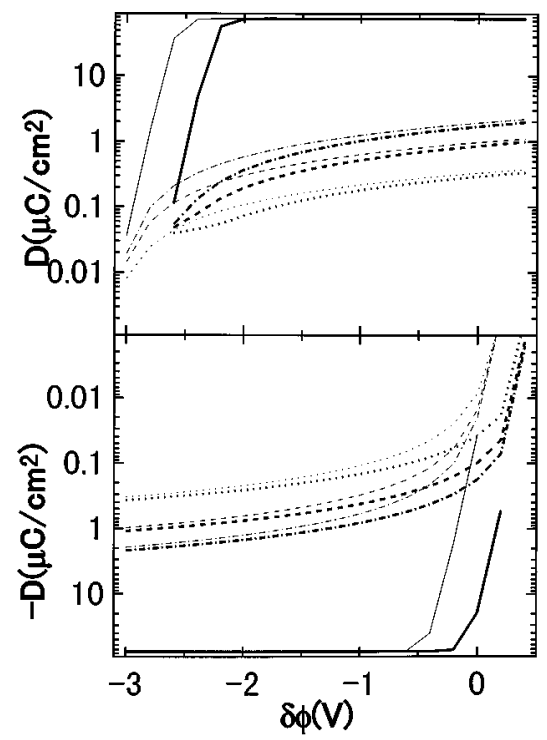

FIG. 10. $\delta \phi$ dependence of $D$ for $l_{f}=100 \mathrm{~nm}, \varepsilon_{f}=1$, and $N_{A f}$ $=10^{18} \mathrm{~cm}^{-3}$ in $\mathrm{PbTiO}_{3} / \mathrm{SiO}_{2} / \mathrm{Si}$ at $25^{\circ} \mathrm{C}$. The thin lines and the thick lines corresponds to $N_{A}=10^{10}$ and $10^{16} \mathrm{~cm}^{-3}$, respectively.

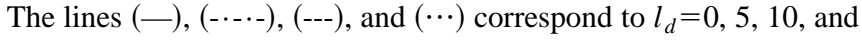
$30 \mathrm{~nm}$, respectively.

mechanism, because it is expected to be not important when the ferroelectric charge is compensated by the space charge, and because experimental results suggest a unidomainlike structure. Before comparing the above results with the experiments, we examine the effect of the multidomain.

The structure in Fig. 16 is a model for a ferroelectric film on insulating substrate with and without a top metallic electrode. Here, we assume only $180^{\circ}$ domain wall and treat the space charge layer in a simple way. ${ }^{34}$ The multidomain is represented by $P(y)=P x$ for $2 a n<y<(2 n+1) a$ and $P(y)$ $=-P x$ for $(2 n \overline{+} 1) a<\bar{y}<2 a(n+1)(x$ : unit vector in the $x$ direction, $n$ : integer).

The free energy $F$ is given by $\left[F_{0}\right.$ is defined in Eq. (2)]

$$
F_{f}^{\prime}=F_{0}+\eta(\nabla P)^{2}+F_{d}^{\prime},
$$
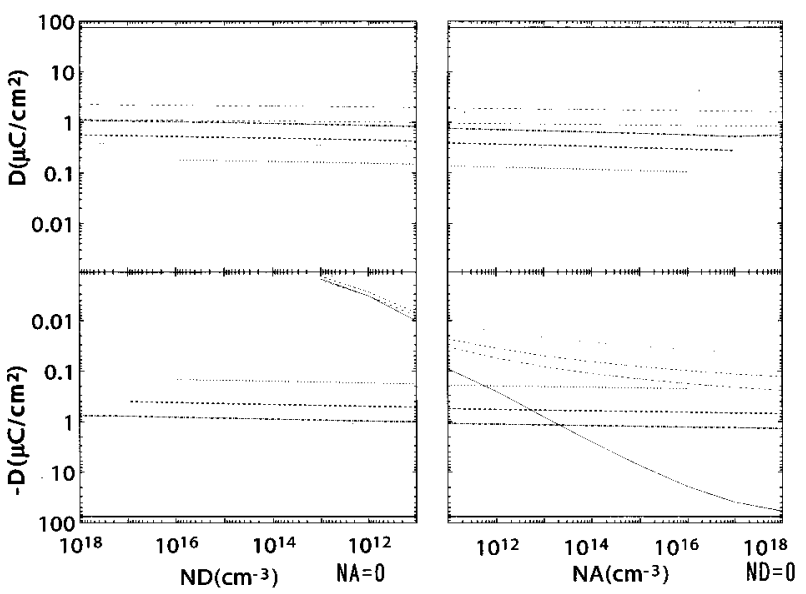

FIG. 11. Doping dependence of $D$ for $l_{f}=100 \mathrm{~nm}, \varepsilon_{f}=1$, and $N_{A f}=10^{18} \mathrm{~cm}^{-3}$ in $\mathrm{PbTiO}_{3} / \mathrm{SiO}_{2} / \mathrm{Si}$ at $25^{\circ} \mathrm{C}$. The thin and the thick lines corresponds to $\delta \phi=0$ and $-1.7 \mathrm{~V}$, respectively. The lines $(-),(-\cdots-),(---)$, and $(\cdots)$ correspond to $l_{d}=0,5,10$, and 30 $\mathrm{nm}$, respectively. 


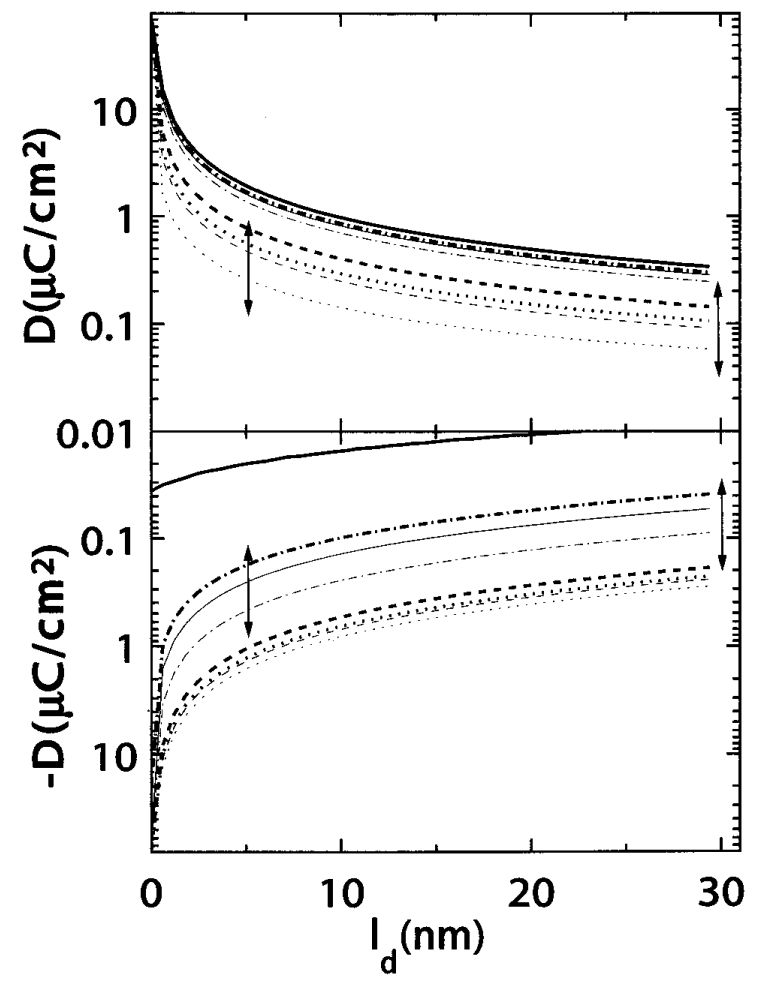

FIG. 12. $l_{d}$ dependence of $D$ for $l_{f}=100 \mathrm{~nm}$ and $\varepsilon_{f}=1$ in $\mathrm{PbTiO}_{3} / \mathrm{SiO}_{2} / \mathrm{Si}$ at $25{ }^{\circ} \mathrm{C}$. The thin and the thick lines corresponds to $N_{A f}=10^{10}$ and $10^{18} \mathrm{~cm}^{-3}$, respectively. The lines (-), (-.-.), $(---)$, and $(\cdots)$ correspond to $\delta \phi=0$ and $N_{A}=10^{10} \mathrm{~cm}^{-3}, \delta \phi=0$ and $N_{A}=10^{16} \mathrm{~cm}^{-3}, \delta \phi=-1.7 \mathrm{~V}$ and $N_{A}=10^{10} \mathrm{~cm}^{-3}, \delta \phi=-1.7 \mathrm{~V}$ and $N_{A}=10^{16} \mathrm{~cm}^{-3}$, respectively. Typical experimental estimates are shown by arrows.

where $F_{d}^{\prime}$ is the depolarization energy.

$F_{d}^{\prime}$ for a stripe multidomain without electrodes is given by using the results for magnetic film ${ }^{35}$ which is reduced by the space-charge layer. The inhomogeneity term gives the domain-wall energy per domain $F_{w} / a=\gamma / a$, where $a$ is the domain width. Details of the derivation and analytical expression of $F_{d}^{\prime}$ and $\gamma$ are described in Appendix E. Minimizing $F_{f}^{\prime}$ with respect to $P$ and $a$, the equilibrium $a$ and $P$ are obtained.

Figure 17 shows the numerical results for $\mathrm{PbTiO}_{3}$, and $\mathrm{BaTiO}_{3}$, where the domain-wall width $2 \xi$ is assumed to be $1.8 \mathrm{~nm}$ for both ferroelectrics. ${ }^{36-38}$ The minimum thickness $l_{f}^{\mathrm{min}}$ to allow the ferroelectric phase without a space-charge layer is $7 \mu \mathrm{m}$ for $\mathrm{BaTiO}_{3}$ and $1 \mu \mathrm{m}$ for $\mathrm{PbTiO}_{3}$. The thickness of the space-charge layer that is used in the literature lies between 10 and $50 \mathrm{~nm}$. With a $10 \mathrm{~nm}$-thick space-charge layer that totally compensates $P_{s}$, the $l_{f}^{\min }$ is $1 \mu \mathrm{m}$ for $\mathrm{BaTiO}_{3}$ and $200 \mathrm{~nm}$ for $\mathrm{PbTiO}_{3}$. With a thicker space-charge layer, $l_{f}^{\min }$ was found to increase, which is not shown in Fig. 17.

The results should be compared with those for the limiting case of $l_{d}=\infty$ in Sec. III. According to Fig. 5 and the analytical results in Sec. II B, $l_{f}^{\mathrm{min}}$ is almost independent of $l_{d}$ for $l_{f}>$ space-charge layer thickness. Therefore, $l_{f}^{\mathrm{min}}$ for $l_{d}$ $=\infty$ is much shorter than the $l_{f}^{\min }$ shown in Fig. 17.

Moreover, the domain size $a$ at $l_{f}^{\mathrm{min}}$ is below $10 \mathrm{~nm}$ in Fig. 17(a), especially a few nm for the ferroelectric with a

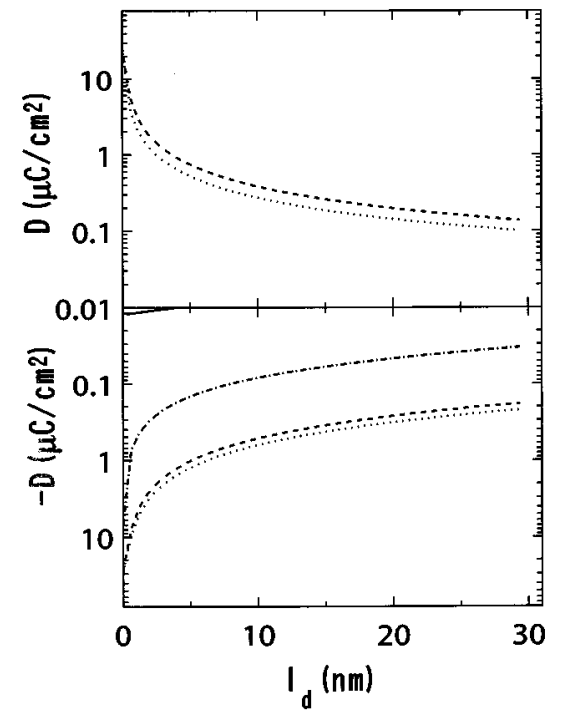

FIG. 13. $l_{d}$ dependence of $D$ for $l_{f}=350 \mathrm{~nm}$, and $N_{A f}$ $=10^{18} \mathrm{~cm}^{-3}$, and $\varepsilon_{f}=1$ in ferroelectric $/ \mathrm{SiO}_{2} / \mathrm{Si}$ at $25^{\circ} \mathrm{C}$. The other parameters are same as those in Fig. 12 except the GLD parameters, which are now those of $\mathrm{BaTiO}_{3}$. For $D>0$, the results only with $\delta \phi=-1.7 \mathrm{~V}$ are shown. $l_{f}=350 \mathrm{~nm}$ is used, because $P_{s}>0$ is unstable below $300 \mathrm{~nm}$. The lines $(-),(-\cdot-)),(--)$, and $(\cdots)$ correspond to the $\delta \phi$ and $N_{A}$ values in Fig. 12.

space-charge layer. Namely, the domain size is close to the domain-wall width. In this case, $x$-ray diffraction should observe a $c$-axis lattice constant averaged over the domain and the wall and yield the appreciably reduced $c$-axis lattice constant. Therefore, the $c$ axis of ferroelectric on an insulator without a top metallic electrode should be shorter than that on a conductor with a top metallic electrode, which is not experimentally observed. Furthermore, x-ray diffraction of epitaxial films on semi-insulating and insulating substrates reveals that they take the ferroelectric phase much below $l_{f}^{\mathrm{min}}$ indicated in Fig. 17. ${ }^{8}$ The results suggest that the inclusion of

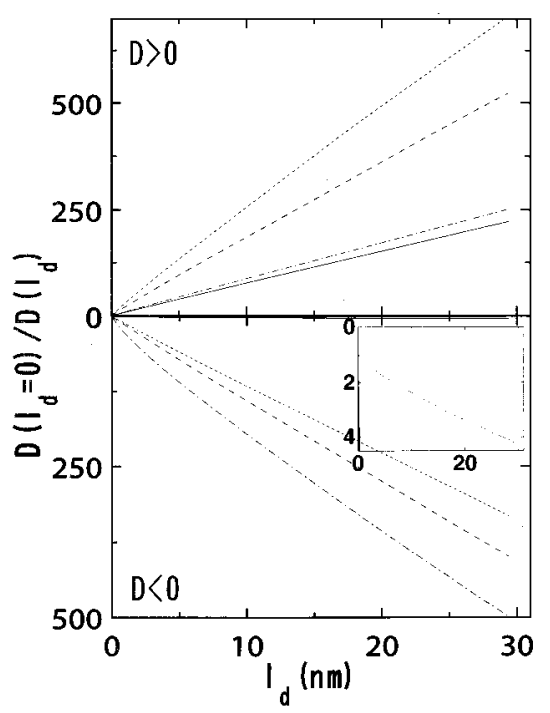

FIG. 14. Replot of Fig. 12 for $N_{A f}=10^{18} \mathrm{~cm}^{-3}$ (thick lines in Fig. 12). The lines (-), (-.-.), (---), and ( $\cdots)$ correspond to the $\delta \phi$ and $N_{A}$ values in caption of Fig. 12, respectively. The inset is a blowup showing the results for $\delta \phi=0$ and $N_{A}=10^{10} \mathrm{~cm}^{-3}$. 


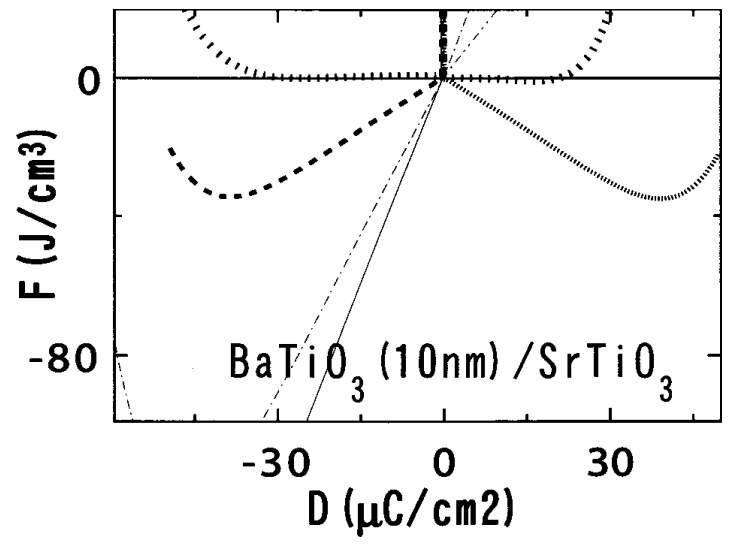

FIG. 15. $F$ vs $D$ for 10 nm-thick $\mathrm{BaTiO}_{3} / n-\mathrm{SrTiO}_{3}$. For $\mathrm{SrTiO}_{3}, \varepsilon_{s}=300, N_{A}=0$ and $N_{D}=10^{16} \mathrm{~cm}^{-3}$. For $\mathrm{BaTiO}_{3}, \varepsilon_{f}=1$, $N_{A}=10^{18} \mathrm{~cm}^{-3}$ and $N_{D}=0$ ( $p$ type : thin lines), and $N_{A}=0$ and $N_{D}=10^{18} \mathrm{~cm}^{-3}$ ( $n$-type: thick lines). The lines $(-),(-\cdots),(-\cdots$ $\cdots),(--),(\cdots)$, and $(\cdots)$ correspond to $\delta \phi=-4,-3$, and $-2 \mathrm{~V}$ ( $p$ type), $-1,0$, and $1 \mathrm{~V}$ ( $n$ type), respectively.

the multidomain effect is not as important as the exact treatment of the space-charge layer as shown in Secs. II and III.

The results in Fig. 17 are understood by regarding the effect of the multidomain as the confinement of the electric flux near the ferroelectric surface region with a thickness of $a$ in the ferroelectric and in the insulator (Fig. 16).

Extending this consideration, we analyze the domain structure in $\mathrm{F} / \mathrm{I} / \mathrm{S}$, when a ferroelectric film is semiconducting and has the space charges. First, the space charges reduce $F_{d}$ far more effectively than multidomain formation, at least, for $l_{d} \leqslant 30 \mathrm{~nm}$. Typically, the reduction is $(\mathcal{D} / P)^{2}<10^{-6}$, which is equivalent to a short $l_{d}$ for which the stable domain size is very large and can be virtually regarded as a unidomain. Viewing differently, the multidomain formation in a spacecharge shielded ferroelectric reduces $F_{d}$ by a factor of 10 for $a / l_{d} \sim 0.1$. According to Eq. (12), the free-energy gain $F_{d}\left(\mathrm{PbTiO}_{3}\right)$ is approximately $\left[0.9 \times\left(0.026 \mathrm{~V} \times 80 \mu \mathrm{C} / \mathrm{cm}^{2}\right.\right.$ $\left.\left.+l_{e}\left(0.1 \mu \mathrm{C} / \mathrm{cm}^{2}\right)^{2} / 2 \varepsilon_{0}\right)\right] / l_{f} \sim 0.1 \mathrm{~J} / \mathrm{cm}^{3} /\left(l_{f} / 200 \mathrm{~nm}\right)$. On the other hand, the free-energy loss by the wall formation for $a$ $=l_{d} / 10 \sim 3 \mathrm{~nm}$ is $70 \mathrm{~J} / \mathrm{cm}^{3}(3 \mathrm{~nm} / a)$.

Therefore, the energy reduced by the multidomain formation is tiny in comparison with the domain-wall energy. Eventually, a large domain that can be virtually regarded as a unidomain is stabilized.

(ii) Stability of the space charge layer in the semiconductor.

The stability of the space-charge layer in F/S and F/M/I/S is discussed in Ref. 13, and we concentrate on the stability in $\mathrm{F} / \mathrm{I} / \mathrm{S}$ in this section. The assumption of semiconductivity in

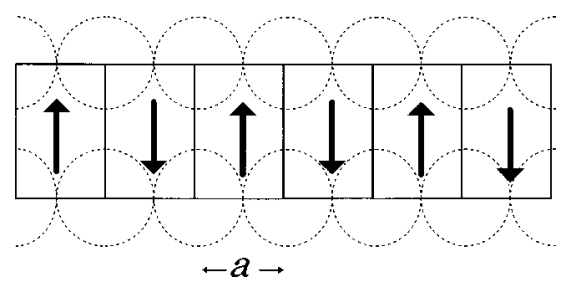

FIG. 16. Model for multidomain calculation. The dotted lines show electric-field lines, and they are confined in the surface with thickness $a$.
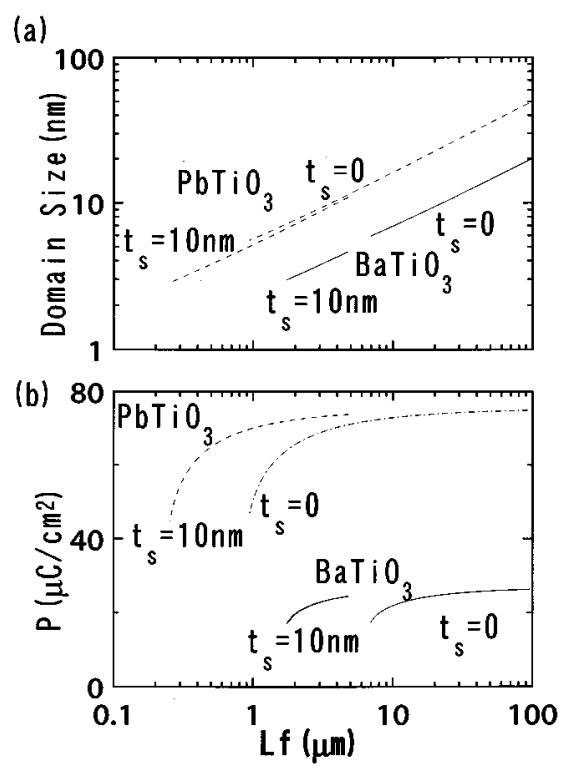

FIG. 17. Domain size width (a) and spontaneous polarization of $\mathrm{PbTiO}_{3}(---)$ and $\mathrm{BaTiO}_{3}(-)$ thin films (b) calculated for given space-charge layer thickness $\left(t_{s}\right)$.

ferroelectrics allows the stability of the ferroelectric phase in $\mathrm{F} / \mathrm{I} / \mathrm{S}$ and ferroelectric/semi-insulator, without introducing the surface state or the defects at the interface. This is consistent with the $\mathrm{x}$-ray observation of the $c$-axis oriented ferroelectric phase in F/I/S. Furthermore, the space charge in the semiconductor induced by $P_{s}$ is experimentally found to be $0.1-1 \mu \mathrm{C} / \mathrm{cm}^{2}$ for an effective $l_{d}=5-10 \mathrm{~nm}$ in agreement with Fig. 12. Moreover, the value of the semiconductor space charge was calculated to be insensitive to the charged trap density as well as the value of $P_{s}$ (Fig. 18). Namely, $D$ is almost the same for the various trap density as long as the charge density at the trap is not same as $P_{s}$. To include the effect of the charged trap in the formulation of Sec. II A, we modified Eq. (6b) to $D=\varepsilon_{0} E_{f}+P+e S$, where $S$ is the charge density per area.

However, $D$ is reported to disappear gradually in typically one hour, except for the result by Sugibuchi et al. This instability appears to contradict the stability of the ferroelectric phase indicated in Sec. III. We tentatively explain the gradual disappearance of the semiconductor space charge by the electric field near the $\mathrm{F} / \mathrm{I}$ interface. The electric field $E_{f}$ at $x=-l_{d}$ (the $\mathrm{F} / \mathrm{I}$ interface) is unphysically large but decreases exponentially with $x$. On the other hand, the electric field in the insulator $E_{d}$ is constant. Therefore, we use $E_{d}$ as a typical measure of the electric field near the F/I interface. $E_{d}$ is given by $\left(\Psi_{f}+\delta \phi-\Psi_{s}\right) / l_{d}$, according to Eq. (7). Figure 4 indicates that $\left|\Psi_{f}+\delta \phi-\Psi_{s}\right|$ is approximately $1.5 \mathrm{~V}$ for $l_{d}$ $=5$ and $30 \mathrm{~nm}$ and not much dependent on $l_{d}$. Consequently, $E_{d}$ decreases with increasing $l_{d}$, e.g., $3 \mathrm{MV} / \mathrm{cm}$ for $l_{d}=5 \mathrm{~nm}$ and $0.5 \mathrm{MV} / \mathrm{cm}$ for $l_{d}=30 \mathrm{~nm}$. The enormous field in the insulator is very likely to move the carrier into the traps, the defects, or the surface states, which reduces the electric flux $D$ flowing into the semiconductor. The filling process of the traps, the defects, or the surface states would proceed gradually with time to compensate $P_{s}$, and finally the space-charge layer in the semiconductor would vanish.

If this is the cause of the disappearance of the semicon- 


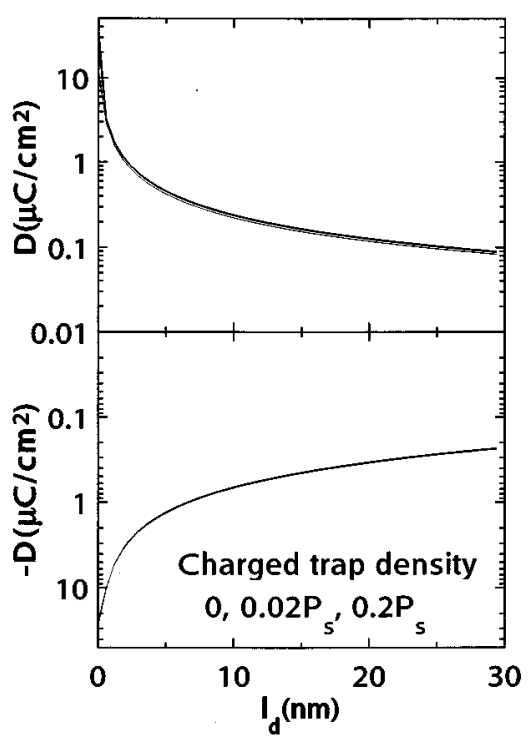

FIG. 18. $D$ vs $l_{d}$ for various values of the charged trap densities at the $\mathrm{F} / \mathrm{I}$ interface $0, \pm 2 \%$, and $\pm 20 \%$ of the spontaneous polarization $\left(P_{s}\right)$ in $\mathrm{PbTiO}_{3} / \mathrm{SiO}_{2} / \mathrm{Si}$ at $25^{\circ} \mathrm{C}$. The parameters are $l_{f}$ $=100 \mathrm{~nm}, \delta \phi=-1.7 \mathrm{~V}$, and $N_{A}=N_{A f}=10^{16} \mathrm{~cm}^{-3}$.

ductor space charge, it can be suppressed by use of a rigid insulator and by increasing its thickness $l_{d}$. The latter is contrary to what is usually believed. However, the longest retention of the semiconductor space charge in the F/I/S structure was indeed achieved using the thickest insulating layer. ${ }^{10}$ In this experiment, $\mathrm{Bi}_{4} \mathrm{Ti}_{3} \mathrm{O}_{12}$ was used. Nontheless, this cannot be the cause of the long retention, because the stability is insensitve to the ferroelectric parameters as suggested by Figs. 12 and 13. Additionally, $D$ estimated from the condunctance modulation ${ }^{10}$ is of order $0.1 \mu \mathrm{C} / \mathrm{cm}^{2}$ in agreement with the result for $l_{d}=30 \mathrm{~nm}$ as in Fig. 12 .

The experimetally observed $D$ can be explained by the interfacial traps or by the $\nabla P$ effect without introducing the semiconductivity of the ferroelectric. For the former, $P_{s}$ becomes monostable, and its stability is unrealsitically too sensitive to the defect density, according to Ref. 13. If the effect of $\nabla P$ mainly determines $D, P_{s}$ crystallograpically estimated should decrease near the F/I interface. This means that the average $P_{s}$ estimated by $\mathrm{x}$-ray diffraction should be significantly reduced in $\mathrm{F} / \mathrm{I} / \mathrm{S}$, if the ferroelectric film is sufficiently thin. However, the ferroelecric in F/I/S has crystallograpic $P_{s}$ as in other heterostructures.

In the present model, the ferroelectric phase in $\mathrm{F} / \mathrm{I} / \mathrm{S}$ is stabilized mainly by the space charge in the ferroelectric. When the polarization swiches between stable polarization states, the space charge must change drastically. Unlike the $\mathrm{F} / \mathrm{S}$, the charge needs to be generated in the ferroelectric or supplied from the metal electrode through the ferroelectric, as long as the insulator layer is insulating. However, the high resistance in the ferroelectric suppresses the current. Here, the net current flowing from the external circuit during the switching is $|D(P>0)-D(P<0)| \times$ net switchng area. Furthermore, the width of the equilibrium $D$ becomes narrow in the F/I/S in comparison with that of the F/S (Fig. 3). These two causes severely limit the simultaneous satisfaction of the switching speed and the retention of $D$. Indeed, the switiching speed of the device reported by Sugibuchi was very low, and, furthermore, no simultaneous achievement of the fast switching and the long retention in the F/I/S was reported so far. This difficulty can be resolved partly by using a very thin ferroelectric, which reduces the net resistance of the ferroelectric, although it tends to be monostable, depending on the GLD parameters. Consequently, this requires a careful choice of the ferroelectric and the preparation process.

(iii) Bistability of ferroelectric polarization on high- $T_{c}$ related materials.

The parent phases of high- $T_{c}$ semiconductors have the charge-transfer band gap of 1.5-2 eV, but most of them can only be doped with either acceptors or donors. Theoretical studies also support these experimental observations. For example, no $n$-type $\mathrm{La}_{2} \mathrm{CuO}_{4}$ has been reported so far. Therefore, the $\mathrm{La}_{2} \mathrm{CuO}_{4}$ surface may only be in the accumulation and the depletion, in contrast with the accumulation and the inversion in an ordinary semiconductor. If $P_{s}$ is monostable on $\mathrm{La}_{2} \mathrm{CuO}_{4}$, i.e., stable only at one polarity, there is nothing to wonder, because it is stabilized by holes in $\mathrm{La}_{2} \mathrm{CuO}_{4}$. However, the ferroelectric switching as well as its conduction modulation of $\mathrm{La}_{2} \mathrm{CuO}_{4}$ that was retained was observed. ${ }^{14,39}$ This suggests that $P_{s}$ is bistable and induces electrons, at least, at some part near the ferroelectric/ $\mathrm{La}_{2} \mathrm{CuO}_{4}$ interface.

The existence of $P_{s}$ in a short $l_{f}$ film for $l_{e}=\infty$ shown in Secs. II B and III assures that $P_{s}$ can exist on a perfect insulator due to the induction of the free carriers in the ferroelectric. Therefore, $P_{s}$ can have a stable polarity that induces the electrons in the ferroelectric near the ferroelectric/ $/ \mathrm{La}_{2} \mathrm{CuO}_{4}$ interface and can be bistable on $\mathrm{La}_{2} \mathrm{CuO}_{4}$.

(iv) Absorption current and the ferroelectric surface.

The free surface and the interface where $P_{s}$ changes abruptly have free charges according to the present theory. This corresponds to the surface space-charge layer suggested by Känzig. ${ }^{2}$ If the surface or the interface are free from disorders and defects, a surface conduction is expected. Namely, ferroelectrics should have conductivity in the surface perpendicular to the direction of $P_{s}$, although it is expected to be low near RT due to the low mobility resulting from a strong electron-phonon coupling. On the other hand, the conductivity perpendicular to the surface should be very low. Moreover, there is a possibility of a $p n$ junction formation near the ferroelectric surface, if the space charge is in the inversion. Therefore, the resistivity along the direction of $P_{s}$ is drastically changed with the switching of the polarization. Possibly related phenomena are observed in the leakage current of the ferroelectric. ${ }^{20,21}$

It is well known that bulk ferroelectrics contain free charges that are emitted during the process of the poling. The resultant current is called as an absorption current. This phenomenon is naturally explained by the space-charge layer in the present model.

Finally, the crutial role of the free charge in narrow-gap ferroelectrics have been well studied. In the present paper, the free charge appears at the ferroelectric surface due to the enoumous depolarization field. Consequently, it screens the electrostatic interaction of the atomic dipoles and, thus, may reduce the spontaneous polarization. This can be a microscopic mechanism of $\nabla P$ at the atomically well-defined surface. This effect was not consistently treated. 


\section{CONCLUSION}

We estimated the stability of the ferroelectric phase and the space-charge layer in a semiconductor, by assuming the ferroelectric as a semiconductor. The ferroelectric treated in this article, e.g., $\mathrm{BaTiO}_{3}$ and $\mathrm{PbTiO}_{3}$, is usually regarded as an insulator. However, the enormous depolarization field at the free surface or at the interface with an insulator requires the compensation of $P_{s}$ by the surface charges. By introducing the semiconductivity, the electric flux $D$ due to $P_{s}$ becomes independent of $P_{s}$. In other words, a material with a small dimension that possesses crystallographic properties of the ferroelectric phase does not intrinsically exhibit some kind of electrical properties of the bulk ferroelectric. Namely, we can predict the crystallographic properties of the ferroelectric except for those of a ultrathin, e.g., $1 \mathrm{~nm}$, ferroelectric by neglecting the depolarization instability (e.g., consider only the stress) but must consider it for the prediction of the electrical flux emitted from the ferroelectric.

To work with this assumption, we have first constructed a band diagram following the procedure of the semiconductor physics. The model could successfully explain the experimentally observed stability of the ferroelectric phase in the $\mathrm{F} / \mathrm{I} / \mathrm{S}$ and the F/I. Moreover, the value of the semiconductor space charge $D$ agrees with the experimental for the $\mathrm{F} / \mathrm{I} / \mathrm{S}$. Furthermore, this value was found to be insensitive to the ferroelectric thickness $l_{f}$, the GLD parameters, i.e., the conventional ferroelectric properties, and the surface states or the charged trap density at the F/I interface. Instead, it is determined mainly by the effective insulator thickness $l_{e}$ and the parameters characterizing the heteroband structure. $D$ was found to be inversely proportional to $l_{e}$ at relatively large $l_{e}$. This numerical result as well as others are well predicted by approximate analytical estimates [Eqs. (16c) and $(16 \mathrm{~d})]$.

The small $D$ values in the F/I/S experimentally observed are usually believed to be limited by the technology such as the defects and a small effective bias across the ferroelectric layer. The theory confirmed that $D$ can be slightly increased by controlling the trap density and the work function of the ferroelectrics. However, the order of magnitude value of $D$ was found to be intrinsically limited by the semiconductor properties of the ferroelectric that suppress the depolarization instability. A ferroelectric field-effect transistor (FET) having a $\mathrm{F} / \mathrm{I} / \mathrm{S}$ should work practically without the limitation of the ferroelectric thickness, e.g., $20 \mathrm{~nm}\left(\mathrm{PbTiO}_{3}\right)$ and $35 \mathrm{~nm}$ $\left(\mathrm{BaTiO}_{3}\right)$, as long as a crystallographically good film with a low defect density is obtained. Furthermore, the limitation of the lateral dimension should also be small because the stability was found without introducing multidomain. Moreover, there is a possibility that a long retention of $D$ can be achieved using the F/I/S structure as well as the F/S, while only one successful result is reported so far to the best of our knowledge. ${ }^{10}$ The short retention is probably due to the filling of the surface states at the ferroelectric/insulator interface and can be reduced by improving the interface or increasing the insulator thickness.

An ultrathin $\mathrm{BaTiO}_{3}$ on $\mathrm{SrTiO}_{3}$ was found to be stable in agreement with experiments. However, it was found that $\mathrm{BaTiO}_{3}$ loses the bistable polarization below $\sim 10 \mathrm{~nm}$ and becomes monostable. Additionally, the mechanism of the switching of ferroelectrics on high- $T_{c}$ superconductors and the related material that may possess holes but no electrons was explained. Finally, the relationship of the present model to the surface effect and the absorption current was briefly discussed.

We may add that the experimental studies of the F/I/S directed toward the realization of a ferroelectric FET can provide a unique and an important opportunity to reveal the ferroelectric size effect by allowing quantitative comparison with theories.

This work was performed under the support through a Grant-in-Aid for Scientific Research from the Ministry of Education, Science, and Sports and the Sumitomo Foundation. The author acknowledges useful discussions with Professor S. Uchida, Professor T. Yamada, and Professor Y. Ishibashi.

\section{APPENDIX A: $\nabla P$ EFFECT}

The $\nabla P$ effect ${ }^{5}$ can be approximated as the effective change of $l_{e}$ and $l_{f}$ to $\left(l_{e}+\xi / 2\right)$ and $\left(l_{f}-\xi / 2\right)$, respectively, and an additional energy $\left(\eta|\nabla P|^{2}\right)$, as shown below. Namely, the $\nabla P$ effect is similar to the surface dead layer. We estimate the effect of $\nabla P \neq 0$ in a single-domained insulating ferroelectric using the symbols defined in the text and Fig. 1. For simplicity, we consider a metal/insulator/ ferroelectric/metal structure. In this case, we drop off $F_{s}$ in Eq. (1), and the term in Eq. (1) affected by the $\nabla P$ effect is

$$
F^{\prime}=\int_{-l_{f}-l_{d}}^{-l_{d}} d x\left(\eta|\nabla P|^{2}+\varepsilon_{0} E_{f}^{2} / 2\right) / l_{f}+l_{e} D^{2} / 2 \varepsilon_{0} l_{f} .
$$

For $\nabla P=0$, using Eqs. (6b) and (7) with $\Psi_{s}=\delta \phi+V_{e}=0$ and $\Psi_{f}=-E_{f} l_{f}$, we have

$$
\begin{gathered}
E_{f}=-l_{e} P / \varepsilon_{0}\left(l_{e}+l_{f}\right), \quad D=l_{f} P /\left(l_{e}+l_{f}\right), \\
F_{1}^{\prime}=l_{e} P^{2} / 2 \varepsilon_{0}\left(l_{e}+l_{f}\right) .
\end{gathered}
$$

For $\nabla P=P / \xi\left(P=0\right.$ at $\left.x=-l_{d}\right)$, we approximate the $P$ distribution by a constant-bulk- $P\left(P_{0}\right.$ : thickness $\left.l_{f}-\xi / 2\right)$ and a zero- $P$ parts (thickness $\xi / 2$ ). Using Eqs. (6b) and (7) with $\Psi_{s}=\delta \phi+V_{e}=0$ and $\Psi_{f}=-E_{f}\left(l_{f}-\xi / 2\right)=D / \varepsilon_{0}(\xi / 2$ $\left.+l_{e}\right)$, we have

$$
\begin{aligned}
E_{f}=- & \left(l_{e}+\xi / 2\right) P_{0} / \varepsilon_{0}\left(l_{f}+l_{e}\right), \quad D=\left(l_{f}-\xi / 2\right) P /\left(l_{e}+l_{f}\right), \\
F_{2}^{\prime}= & \eta\left(P_{0} / \xi\right)^{2} \xi / l_{f}+\left[\left(l_{f}-\xi / 2\right) \varepsilon_{0} E_{f}^{2} / 2\right. \\
& \left.+(\xi / 2) D^{2} / 2 \varepsilon_{0}\right] / l_{f}+l_{e} D^{2} / 2 \varepsilon_{0} l_{f} \\
= & \eta P_{0}^{2} / \xi l_{f}+\left(1-\xi / 2 l_{f}\right)\left(l_{e}+\xi / 2\right) P_{0}^{2} / 2 \varepsilon_{0}\left(l_{e}+l_{f}\right) .
\end{aligned}
$$

The above expressions for $E_{f}$ and $D$ are also derived easily by applying Eq. (6) of Ref. 5 to the present model. The difference of $F_{1}^{\prime}$ and $F_{2}^{\prime}$ is

$F_{2}^{\prime}-F_{1}^{\prime}=P_{0}^{2}\left[\eta / \xi l_{f}+l_{e}\left(\xi / l_{e}-\xi / l_{f}-\xi^{2} / 2 l_{e} l_{f}\right) / 4 \varepsilon_{0}\left(l_{e}+l_{f}\right)\right]$.

The condition $\xi \gg l_{f}$ is essentially the same as $\nabla P=0$. Therefore, we consider two limiting cases of $\xi \ll l_{f}$. For $l_{e}$ $\ll l_{f}$, we have $F_{2}^{\prime}-F_{1}^{\prime} \approx P_{0}^{2}\left(\eta / \xi+\xi / 4 \varepsilon_{0}\right) l_{f}$, which is always positive. This suggests that the $\nabla P$ effect is not effective for 
suppressing the depolarization instability but may slightly modify the results in the text for a very thin $l_{e}$.

For $l_{e} \gg l_{f}$, we have $F_{2}^{\prime}-F_{1}^{\prime} \approx P_{0}^{2}\left(\eta / \xi-\xi / 4 \varepsilon_{0}\right) / l_{f}$. Here, $\eta|\nabla P|^{2}$ is usually much smaller than the bulk free energy density of the ferroelectric phase, and $P_{0}^{2} \xi / 4 \varepsilon_{0}$ is much smaller than $l_{e} D^{2} / 2 \varepsilon_{0}$ for $l_{e} \gg l_{f}$. Therefore, $F_{2}^{\prime}-F_{1}^{\prime}$, i.e., the $\nabla P$ effect, is unimportant for a thick $l_{e}$.

\section{APPENDIX B: ANALYSIS OF DOMAIN STRUCTURE IN THE F/I/S}

High-frequency capacitance-voltage $(C-V)$ curves of several $\mathrm{F} / \mathrm{I} / \mathrm{S}$ structures are reported. Some of them exhibited $C$ - $V$ hysteresis corresponding to the ferroelectric polarization reversal, although they disappeared in a short period. The ratio of two capacitance for two ferroelectric polarization states are approximately $0.1{ }^{11,12}$

Let us analyze these results. Suppose that a $\mathrm{BaTiO}_{3}$-type insulating ferroelectric film forms a multidomain having a domain width, e.g., $5 \mathrm{~nm}$ much wider than the insulator thickness, e.g., $2 \mathrm{~nm}$. Then, we may crudely model each domain and the semiconductor space-charge layer underneath it as uniform. For a $c$-axis oriented film as used in most experiments, the polarization of the unidomain would orient perpendicular to the film surface. Accordingly, the surface charge in each unidomain is defined as $P^{+},-P^{-}, 0$.

The capacitance $C_{i}$ defined for a unidomain per unit area is

$$
1 / C_{i}=1 / C_{i, s}+1 / C_{i \text {,insulator }}+1 / C_{i, \text { ferroelectric }},
$$

where $C_{i \text {,insulator }}, C_{i \text {,ferroelectric }}$, and $C_{i, s}$ are capacitance of the insulator, the ferroelectric, and the semiconductor spacecharge layer per unit area, respectively. The equation is rewritten as

$$
\begin{gathered}
C_{i}=C_{i, \text { in }}^{\prime} /\left(1+C_{i, \text { in }}^{\prime} / C_{i, s}\right), \\
1 / C_{i, \text { in }}^{\prime}=1 / C_{i, \text { insulator }}+1 / C_{i, \text { ferroelectric }},
\end{gathered}
$$

where $C_{i, \text { in }}^{\prime}$ is capacitance of ferroelectric/insulator per unit area. The total capacitance $C_{\text {total }}$ is

$$
C_{\text {total }}=\sum_{i:+ \text { domain }} S_{i} C_{i}+\sum_{i:- \text { domain }} S_{i} C_{i}+\sum_{i: 0 \text { domain }} S_{i} C_{i}
$$

where the first, the second, and the third sums (i) are taken over the domains having $P^{+},-P^{-}$, and 0 polarization, respectively, and $S_{i}$ is the area of each domain. $C_{i}$ for given $P$ values $\left(=P^{+},-P^{-}, 0\right)$ should be the same, and we denote them as $c^{+}, c^{-}$, and $c^{0}$, respectively. By substituting $C_{s}^{+-0}$ into $C_{i, s}$ and $C_{\text {in }}^{\prime}$ into $C_{i, \text { in }}^{\prime}$, we express them as

$$
\begin{gathered}
c^{+}=C_{\mathrm{in}}^{\prime} /\left(1+C_{\mathrm{in}}^{\prime} / C_{, s}^{+}\right) \quad \text { for } P=P^{+}, \\
c^{-}=C_{\mathrm{in}}^{\prime} /\left(1+C_{\mathrm{in}}^{\prime} / C_{, s}^{-}\right) \quad \text { for } P=-P^{-}, \\
c^{0}=C_{\mathrm{in}}^{\prime} /\left(1+C_{\mathrm{in}}^{\prime} / C_{, s}^{0}\right) \quad \text { for } P=0 .
\end{gathered}
$$

Using these expressions, we have

$$
C_{\text {total }}=S^{+} c^{+}+S^{-} c^{-}+S^{0} c^{0},
$$

where $S^{+}, S^{-}$, and $S^{0}$ denotes the net area of domain having $P^{+},-P^{-}$, and 0 , respectively. To simplify the analysis, we analyze the experiments that used a nearly-c-axis oriented ferroelectric, and we neglect the last term in the above equation. Therefore we have,

$$
C_{\text {total }}=C_{\mathrm{in}}^{\prime} S^{+} /\left(1+C_{\mathrm{in}}^{\prime} / C_{, s}^{+}\right)+C_{\mathrm{in}}^{\prime} S^{-} /\left(1+C_{\mathrm{in}}^{\prime} / C_{, s}^{-}\right) .
$$

We can assume that $C^{+}$and $C^{-}$correspond to the accumulation and the inversion conditions of semiconductor surface carrier, respectively, without losing generality. Therefore, we have the inequalities, $C_{s}^{+} \gg C_{s}^{-}$and $C_{s}^{+} \gg C_{\text {in }}^{\prime}$. The above equation is rewritten as

$$
C_{\text {total }}=C_{\mathrm{in}}^{\prime}\left[s^{+} /\left(1+C_{\mathrm{in}}^{\prime} / C_{, s}^{+}\right)+\left(1-s^{+}\right) /\left(1+C_{\mathrm{in}}^{\prime} / C_{, s}^{-}\right)\right] S,
$$

where $S$ is the total capacitance area, and $s^{+}$and $s^{-}$are the area ratios, $S^{+} / S$ and $S^{-} / S$.

The ratio of two values corresponding to different domain configurations specified by the values $s^{+}, s^{+1}$ and $s^{+2}$ is

$$
\begin{aligned}
& {\left[s^{+1} /\left(1+C_{\mathrm{in}}^{\prime} / C_{, s}^{+}\right)+\left(1-s^{+1}\right) /\left(1+C_{\mathrm{in}}^{\prime} / C_{, s}^{-}\right)\right] /\left[s^{+2} /(1\right.} \\
&+\left.\left.C_{\mathrm{in}}^{\prime} / C_{, s}^{+}\right)+\left(1-s^{+2}\right) /\left(1+C_{\mathrm{in}}^{\prime} / C_{, s}^{-}\right)\right] \\
& \sim {\left[s^{+1}+\left(1-s^{+1}\right) /\left(1+C_{\mathrm{in}}^{\prime} / C_{, s}^{-}\right)\right] /\left[s^{+2}\right.} \\
&\left.+\left(1-s^{+2}\right) /\left(1+C_{\mathrm{in}}^{\prime} / C_{, s}^{-}\right)\right] .
\end{aligned}
$$

If $C_{\mathrm{in}}^{\prime} \gg C_{s,}^{-}$, this reduces to $\left(1-s^{+1}\right) /\left(1-s^{+2}\right)$. If $C_{\text {in }}^{\prime}=C_{, s}^{-}$, this reduces to $\left(1+s^{+1}\right) /\left(1+s^{+2}\right) \geqslant 1 / 2$. If $C_{\mathrm{in}}^{\prime} \ll C_{s}^{-}$, this reduces to 1 .

In most experiments, we have the ratio of the minimum capacitance to the maximum of about 0.1 . The above results show that we should assume $C_{\mathrm{in}}^{\prime} \gg C_{, s}^{-}$. Consequently, we have $\left(1-s^{+1}\right) /\left(1-s^{+2}\right) \sim 0.1$. This means the condition on $s^{+1}, s^{+1}=0.9+0.1 s^{+2} \geqslant 0.9$. On the other hand, we expect $s^{+1} \sim s^{+2} \sim 0.5$, if the ferroelectric depolarization instability is eliminated by multidomain formation. This means that $C-V$ data suggest that there should exist a unidomain, or a multidomain very close to a unidomain in $c$-axis oriented ferroelectric/insulator/semiconductor structures.

\section{APPENDIX C: DERIVATION OF EQ. (1)}

For the estimation of the free energy for Fig. 1 we need to evaluate the GL free energy under the external field $E_{f}$ consistently with the electrostatic energy in the insulator $(I)$ and the semiconductor $(S)$. The effect of an external field in a general GL theory is described by the perturbing operator in the Hamiltonian

$$
H=-\zeta h,
$$

where $\zeta$ and $h$ are the order parameter and the field strength, respectively. If the order parameter is the charge density $\rho$, we have $H=\rho \Psi$, where $\Psi$ is the electric potential. The dipole $\underline{p}$ is the sum of a couple of charges $\pm p / l$ locating at $\pm l / 2$. Their $H=\rho \Psi$ in the limit of short $l$ is

$$
\begin{gathered}
{[p \Psi(\underline{l} / 2)-p \Psi(-\underline{l / 2})] / l \approx p[\underline{l} \cdot \nabla \Psi(0)] / l=\underline{p} \cdot \nabla \Psi(0)} \\
=-\underline{p} \cdot \underline{E}
\end{gathered}
$$


Therefore, $H=-\underline{p} \cdot \underline{E}$, where $\underline{E}$ is the electric field. If $E$ is dependent on $\underline{p}$, it is generalized as

$$
-\int \underline{E} \cdot d \underline{p} .
$$

This term is expressed by the field energy, because it is the electrostatic field energy of two charges.

We show a simple example of two expressions. The total dielectric energy is transformed by using relation $P=(\varepsilon$ $-1) \varepsilon_{0} E=\chi \varepsilon_{0} E$,

$$
\begin{aligned}
\varepsilon \varepsilon_{0} \int d v E^{2} / 2 & =\int d v P E / 2+\varepsilon_{0} \int d v E^{2} / 2 \\
& =\int d v P^{2} / 2 \chi \varepsilon_{0}+\varepsilon_{0} \int d v E^{2} / 2,
\end{aligned}
$$

where $\chi$ and $\varepsilon_{0}$ are the susceptibility and the vacuum permittivity, and the integration is performed over the entire space. The first term is the bulk free energy of the dielectric, and the second term is the pure electrostatic field energy. Namely, we have

$$
=F_{0}+\varepsilon_{0} \int d v E^{2} / 2 \quad\left(=F_{0}+\int d v P^{2} / 2 \varepsilon_{0}\right) .
$$

The formula in the parentheses is for an infinite dielectric plate, where $E_{f}$ is caused by the depolarization field ( $D$ $\left.=\varepsilon_{0} E_{f}+P=0\right)$. The equation is same as the conventional charge-potential expression $F_{0}-\int d v\left[\int_{0}^{P} d p E_{f}\right]$ with $E_{f}$ $=-p / \varepsilon_{0}$, where the first and the last terms are the bulk free energy and the depolarization energy. The field expression and the charge-potential expression of the depolarization energy yield the same results.

As a next example, we consider the charge-potential energy and the field energy in $\mathrm{F} / \mathrm{I} / \mathrm{S}$ of a $l_{f}$-thick homogeneous ferroelectric in Fig. 1. An electrode with Ohmic contact is assumed at $x=-l_{d}-l_{f}$. We consider an infinitesimal change of dipole moment $p, \delta p$, where $p$ and $\delta p$ are directed perpendicular to the interface, and $p$ corresponds to $P$. The charges are only in $S$ and $F$. Therefore, the infinitesimal change of electrostatic energy in $S(0 \leqslant x \leqslant \infty)$ and $F\left(-l_{d}\right.$ $\left.-l_{f}<x \leqslant-l_{d}\right)$ is

$$
\begin{aligned}
& \int_{0}^{\infty} d x \delta \rho(x) \Psi(x)-\int_{-l_{d}-l_{f}}^{-l_{d}} d x E(x) \delta p \\
& \quad+\int_{-l_{d}-l_{f}}^{-l_{d}} d x \delta \rho(x) \Psi(x) \\
& =\left[\varepsilon_{s} \varepsilon_{0} \delta E(x) \Psi(x)\right]_{0}^{\infty}+\left[\varepsilon_{0} \delta E(x) \Psi(x)\right]_{l_{d}-l_{f}}^{-l_{d}} \\
& \quad+\varepsilon_{s} \varepsilon_{0} \int_{0}^{\infty} d x \delta E(x) E(x)+\varepsilon_{0} \int_{-l_{d}-l_{f}}^{-l_{d}} d x \delta E(x) E(x) \\
& \quad-\int_{-l_{d}-l_{f}}^{-l_{d}} d x E(x) \delta p,
\end{aligned}
$$

where $\Psi \equiv \Psi(x, p), \quad E \equiv E(x, p), \quad \rho(p) \equiv \rho(x, p), \varepsilon_{s}$ are the potential, the electric field, the charge density, and the dielectric constant of $S$, and $\delta E$ and $\delta \rho$ are the changes by $\delta p$ of $E$ and $\rho$, respectively. The potentials at $x=0$ and $x=\infty$ are

$$
\begin{gathered}
\Psi(0)=-D\left(-l_{d}\right) l_{e} / \varepsilon_{0}+\Psi\left(-l_{d}\right), \quad \Psi(\infty)=0, \\
\Psi\left(-l_{d}-l_{f}\right)=\delta \phi+V e .
\end{gathered}
$$

Using these relations and $\varepsilon_{s} \varepsilon_{0} \delta E(0)=\delta D\left(-l_{d}\right)=\delta p$ $+\varepsilon_{0} \delta E\left(-l_{d}\right)$, Eq. (C1) is

$$
\begin{aligned}
= & -\delta D\left(-l_{d}\right)\left[-D\left(-l_{d}\right) l_{e} / \varepsilon_{0}+\Psi\left(-l_{d}\right)\right] \\
& +\left[\varepsilon_{0} \delta E\left(-l_{d}\right) \Psi\left(-l_{d}\right)-\varepsilon_{0} \delta E\left(-l_{d}-l_{f}\right) \Psi\left(-l_{d}-l_{f}\right)\right] \\
& +\varepsilon_{s} \varepsilon_{0} \int_{0}^{\infty} d x \delta E(x) E(x)+\varepsilon_{0} \int_{-l_{d}-l_{f}}^{-l_{d}} d x \delta E(x) E(x) \\
& +\delta p\left(\Psi\left(-l_{d}\right)-\Psi\left(-l_{d}-l_{f}\right)\right) \\
= & \delta\left[\varepsilon_{s} \varepsilon_{0} \int_{0}^{\infty} d E^{2}(x) / 2+\varepsilon_{0} \int_{-l_{d}-l_{f}}^{-l_{d}} d x E^{2}(x) / 2\right. \\
& \left.+D^{2} l_{d} / 2 \varepsilon_{d} \varepsilon_{0}-D\left(-l_{d}-l_{f}\right) \Psi\left(-l_{d}-l_{f}\right)\right] \\
\approx & \delta\left[\varepsilon_{s} \varepsilon_{0} \int_{0}^{\infty} d x E^{2}(x) / 2+\varepsilon_{0}\right]_{-\infty}^{-l_{d}} d x E^{2}(x) / 2 \\
& \left.+D^{2} l_{d} / 2 \varepsilon_{d} \varepsilon_{0}-p(\delta \phi+V e)\right]^{,}
\end{aligned}
$$

where $\delta[]$ means the change of the value in [] due to $\delta p$. The last expression is the change of the field energy stored in $S, I$, and $F$ due to $\delta p$. The last quasiequality is valid for $l_{f} \gg$ the space charge layer width of the ferroelectric.

The final relation is obtained by integrating the first and the last expressions from $p=0$ to $p=P$ and discarding the terms for $p=0$, because we are interested in the $P$ dependence.

\section{APPENDIX D: EXPRESSIONS OF $\Psi_{s}$ AND $\Psi_{f}$}

For a $p$-type ferroelectric,

$$
\begin{gathered}
\Psi_{f}=2 \beta^{-1} \ln \left[\sqrt{\left(p_{0} / 2 n_{0}\right)}|P-D| / \mathcal{D}\right] \quad \text { strong inversion, } \\
\Psi_{f}=\beta^{-1}((P-D) / \mathcal{D})^{2} / 2+\beta^{-1}
\end{gathered}
$$

weak inversion and depletion,

$$
\Psi_{f}=-\beta^{-1}(P-D) / \mathcal{D}
$$

weak depletion and weak accumulation,

$$
\Psi_{f}=-2 \beta^{-1} \ln (|P-D| / \sqrt{2} \mathcal{D}) \quad \text { strong accumulation. }
$$

For a $p$-type semiconductor,

$$
\Psi_{s}=2 \beta^{-1} \ln \left[\sqrt{\left(p_{0} / 2 n_{0}\right)}|D| / \mathcal{D}\right] \quad \text { strong inversion, }
$$

$\Psi_{s}=\beta^{-1}(D / \mathcal{D})^{2} / 2+\beta^{-1} \quad$ weak inversion and depletion,

$$
\Psi_{s}=-\beta^{-1} D / \mathcal{D}
$$

weak depletion and weak accumulation,

$$
\Psi_{s}=-2 \beta^{-1} \ln (|D| / \sqrt{2} \mathcal{D}) \quad \text { strong accumulation. }
$$


$\mathcal{D}$ is defined in the text by Eq. (14).

\section{APPENDIX E: MULTIDOMAIN EFFECT} by

$F_{f}^{\prime}$ for a ferroelectric film having a thickness $l_{f}$ is given

$$
F_{f}^{\prime}=F_{0}+\eta(\nabla P)^{2}+F_{d}^{\prime}=F_{0}+F_{w} / a+F_{d}^{\prime},
$$

where the lateral dimension of ferroelectric is much larger than the domain size $a$. If the space-charge layer exists having a thickness $t, F_{d}$ is modified from $1.7 \mathrm{P} a / l_{f}$ to

$$
1.7 \mathrm{P}^{2} a t / l_{f}^{2}(\mathrm{esu})
$$

for a homogeneous space-charge layer. ${ }^{34}$ The domain-wall energy $F_{w}$ per domain-wall area is, ${ }^{37}$

$$
\begin{aligned}
F_{w} & =\int_{-\infty}^{\infty}\left[F_{f}\left(P(x)-F_{f}(P)\right)\right] d x \\
& =\left(-2 \alpha_{1} P^{2}-8 / 3 \alpha_{11} P^{4}-46 / 15 \alpha_{111} P^{6}\right) \xi+4 \eta P^{2} / 3 \xi,
\end{aligned}
$$

where $P(x)$ is assumed as $P \tanh (x / \xi)$. The half domain width $\xi$ that minimizes domain-wall energy is given by $\partial \gamma /$ $\partial \xi=0$. This is satisfied for

$$
\xi=\sqrt{4 \eta / 3\left(-2 \alpha_{1} P^{2}-8 / 3 \alpha_{11} P^{4}-46 / 15 \alpha_{111} P^{6}\right)},
$$

yielding the minimum $F_{w}=\gamma=8 \eta P^{2} / 3 \xi$. The material constant $\eta$ is estimated by equating $\xi$ with the experimentally determined half domain width in a bulk ferroelectric. The expressions of $\gamma$ and $\xi$ give the wall energy for an arbitrary $l_{f}$.
${ }^{1}$ K. Kristoffel and P. Konsin, Ferroelectrics 6, 3 (1973).

${ }^{2}$ W. Känzig, Phys. Rev. 98, 549 (1955).

${ }^{3}$ K. Okazaki, H. Igarashi, K. Nagata, and A. Hasegawa, Ferroelectrics 7, 153 (1974).

${ }^{4}$ T. C. Lubensky and M. H. Rubin, Phys. Rev. B 12, 3885 (1975).

${ }^{5}$ W. R. Kretschmer and K. Binder, Phys. Rev. B 20, 1065 (1979); K. Binder, Ferroelectrics 35, 99 (1981).

${ }^{6}$ D. R. Tilley and B. Zeks, Solid State Commun. 49, 823 (1984).

${ }^{7}$ X. L. Zhong, B. D. Qu, P. L. Zhang, and Y. G. Wang, Phys. Rev. B 50, 12375 (1994).

${ }^{8}$ Y. Watanabe, Y. Matsumoto, H. Kunitomo, M. Tanamura, and E. Nishimoto, Jpn. J. Appl. Phys., Part 1 33, 5182 (1994).

${ }^{9} \mathrm{~K}$. Wasa (private communication).

${ }^{10}$ K. Sugibuchi, Y. Kurogi, and N. Endo, J. Appl. Phys. 46, 2877 (1975).

${ }^{11}$ T. Hirai, K. Teramoto, T. Nishi, T. Goto, and Y. Tarui, Jpn. J. Appl. Phys., Part 1 33, 5219 (1994); T. Hirai, K. Teramoto, K. Nagashima, H. Koike, and Y. Tarui, ibid. 34, 4163 (1995).

${ }^{12}$ E. Tokumitsu, K. Itani, B. Moon, and H. Ishihara, J. Appl. Phys. 34, 5202 (1995).

${ }^{13}$ Y. Watanabe, J. Appl. Phys. (to be published).

${ }^{14}$ Y. Watanabe, Appl. Phys. Lett. 66, 1770 (1995); Y. Watanabe, T. Tanamura, and Y. Matsumoto, Jpn. J. Appl. Phys., Part 1 35, 1564 (1996).

${ }^{15}$ V. B. Sandomirskii, Sh. S. Khalilov, and E. V. Chenskii, Sov. Phys. Semicond. 16, 279 (1982).

${ }^{16}$ B. M. Vul, G. M. Guro, and I. I. Ivanchik, Sov. Phys. Semicond. 4, 128 (1970); G. M. Guro, I. I. Ivanchik, and N. F. Kovtonyuk, Sov. Phys. Solid State 11, 1574 (1970).

${ }^{17}$ I. P. Batra, P. Wurfel, and B. D. Silverman, Phys. Rev. B 8, 3257 (1973); P. Wurfel and I. P. Batra, ibid. 8, 5126 (1973).

${ }^{18}$ I. P. Batra, P. Wurfel, and B. D. Silverman, Phys. Rev. Lett. 30, 384 (1973).

${ }^{19}$ G. R. Fox and S. B. Krupanidhi, J. Appl. Phys. 74, 1949 (1993); C. Sudhama, A. C. Campbell, P. D. Maniar, R. E. Jones, R. Moazzami, and C. J. Mogab, ibid. 75, 1014 (1994); R. C. Neville and B. Hoeneisen, ibid. 46, 350 (1975).

${ }^{20}$ P. W. M. Blom, R. M. Wolf, J. F. M. Cillessen, and M. P. C. M. Krijn, Phys. Rev. Lett. 73, 2107 (1994).

${ }^{21}$ Y. Watanabe, Y. Matsumoto, M. Tanamura, H. Asami, and A. Kato, Physica C 235-240, 739 (1994); Y. Watanabe, Appl. Phys. Lett. 66, 28 (1995).
${ }^{22}$ A. J. Bell, Ferroelectrics Lett. 15, 133 (1993).

${ }^{23}$ T. Yamamoto, Integr. Ferroelectr. 12, 161 (1996).

${ }^{24}$ P. Güthner and K. Dransfeld, Appl. Phys. Lett. 61, 1139 (1992); K. Frank, J. Besold, W. Haessler, and C. Seegebarth, Surf. Sci. Lett. 302, L283 (1994).

${ }^{25}$ A. F. Devonshire, Philos. Mag. 40, 1065 (1951).

${ }^{26}$ M. J. Haun, E. Furman, S. J. Jang, H. A. Kinstry, and L. E. Cross, J. Appl. Phys. 62, 3331 (1987).

${ }^{27}$ T. Yamamoto and H. Matsuoka, Jpn. J. Appl. Phys., Part 1 33, 5317 (1994)

${ }^{28}$ B. E. Deal, E. W. Snow, and C. A. Mead, J. Phys. Chem. Solids 27, 1873 (1966); W. M. Werner, Solid-State Electron. 17, 769 (1974).

${ }^{29}$ S. M. Sze, Physics of Semiconductor Devices (Wiley, New York, 1981).

${ }^{30}$ R. L. Anderson, Solid-State Electron. 5, 341 (1961).

${ }^{31}$ W. G. Oldham and A. G. Milnes, Solid-State Electron. 7, 153 (1964).

${ }^{32}$ G. Heiland, Z. Phys. 148, 28 (1957); P. E. Bloomfield, I. Lefkowitz, and A. D. Aronoff, Phys. Rev. B 4, 974 (1971).

${ }^{33}$ G. W. Dietz, W. Antpöhler, M. Klee, and R. Waser, J. Appl. Phys. 78, 6113 (1995); P. F. Baude, C. Ye, and D. L. Polla, Appl. Phys. Lett. 64, 2670 (1994).

${ }^{34}$ W. Y. Shih, W. H. Shih, and I. A. Aksay, Phys. Rev. B 50, 15575 (1994).

${ }^{35}$ C. Kittel, Rev. Mod. Phys. 21, 541 (1949); S. Chikazumi, Physics of Ferromagnetism (Syokabo, Tokyo, 1984), Vol. 2, p. 193 (in Japanese).

${ }^{36}$ E. Jona and G. Shirane, Ferroelectric Crystals (Pergamon, New York, 1962); W. Känzig, in Solid State Physics 4, edited by E. Seitz and D. Turnbull (Academic, New York, 1957).

${ }^{37}$ M. E. Lines and A. M. Glass, Principles and Applications of Ferroelectric and Related Materials (Oxford University Press, Oxford, 1977).

${ }^{38}$ V. A. Zirnov, Zh. Eksp. Teor. Fiz. 35, 1175 (1959) [Sov. Phys. JETP 35, 822 (1959)]; L. N. Bulaevskii, Sov. Phys. Solid State 5, 2329 (1964); J. Padilla, W. Zhong, and D. Vanderbilt, Phys. Rev. B 53, 5969 (1996); M. Tanaka and G. Honjo, J. Phys. Soc. Jpn. 19, 954 (1964); W. J. Merz, Phys. Rev. 95, 690 (1954); J. Fousek and M. Safrakova, Jpn. J. Appl. Phys. 4, 403 (1965).

${ }^{39}$ Y. Watanabe, Y. Matsumoto, and M. Tanamura, Jpn. J. Appl. Phys., Part 1 34, 5254 (1995). 